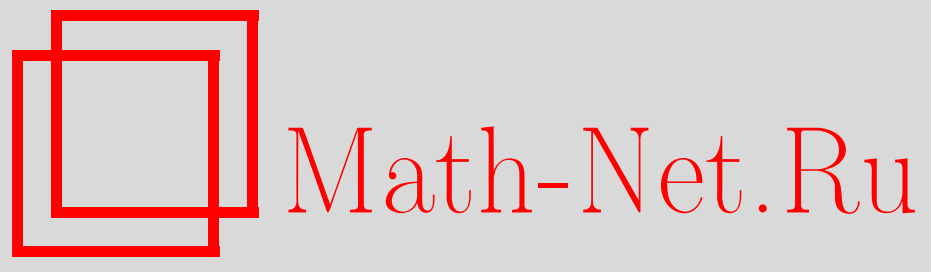

М. Фарбер, Р. Гейган, Д. Шютц, Замкнутые 1-формы в топологии и геометрической теории групп, УМH, 2010, том 65, выпуск 1, 145-176

DOI: https://doi.org/10.4213/rm9345

Использование Общероссийского математического портала Math-Net.Ru подразумевает, что вы прочитали и согласны с пользовательским соглашением http://www . mathnet.ru/rus/agreement

Параметры загрузки:

IP : 54.198 .64 .247

26 апреля 2023 г., 06:37:39

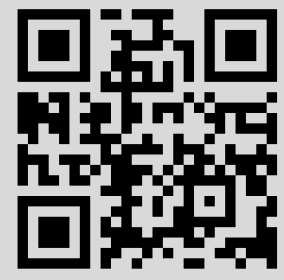




\title{
Замкнутые 1-формы в топологии и геометрической теории групп
}

\begin{abstract}
М. Фарбер, Р. Гейган, Д. Шютц
В настоящей статье мы описываем связи между топологией замкнутых 1-форм и теоретико-групповыми инвариантами Бьери-Неймана-Штребеля-Ренца. Начиная с обзора, мы обобщаем эти сигма-инварианты на конечные клеточные комплексы и показываем, что многие свойства из теоретико-групповой версии имеют аналоги. В частности, мы устанавливаем связь между сигма-инвариантами и свойствами конечности некоторых бесконечнолистных накрытий. Мы также обсуждаем применения этих инвариантов к категории Люстерника-Шнирельмана для замкнутых 1-форм и к существованию несингулярной замкнутой 1-формы в заданном классе когомологий на замкнутых многообразиях высокой размерности.

Библиография: 32 названия.
\end{abstract}

Ключевые слова: сигма-инварианты, кольцо Новикова, подвижность гомологических классов, категория Люстерника-Шнирельмана.

\section{СОДЕРЖАНИЕ}

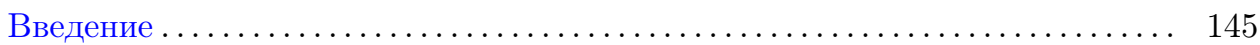

1. Инварианты Бьери-Неймана-Штребеля-Ренца . . . . . . . . . . . . . . . . . 147

2. Сигма-инварианты клеточных комплексов .................. 152

3. Кольцо Новикова и гомологии . . . . . . . . . . . . . . . . . . 157

4. Связь с категорией Люстерника-Шнирельмана для замкнутых 1-форм 158

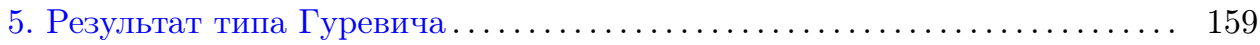

6. Свойство функториальности . . . . . . . . . . . . . . . . . . . . 160

7. Результаты доминирования для накрывающих пространств . . . . . . . 161

8. Подвижность гомологических классов . . . . . . . . . . . . . . 169

9. Функциональное пространство путей в бесконечность . . . . . . . . . 170

Приложение А. Сигма-инварианты цепных комплексов . . . . . . . . . . . 174

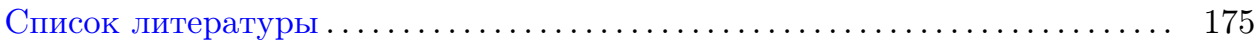

\section{Введение}

В течение трех последних десятилетий наблюдается все возрастающий интерес к топологии замкнутых 1-форм. Начало было положено С. П. Новиковым в [1], [2], где была введена техника теории Морса для изучения классических 
проблем математической физики. По аналогии с комплексом Морса-Смейла для функции Морса на замкнутом многообразии, им был построен цепной комплекс, ныне называемый комплексом Морса-Новикова, ассоциированный с замкнутой 1-формой с невырожденными в смысле Морса особенностями. Хотя для Новикова интерес представляли проблемы уравнений типа Кирхгофа [2]-[4], вскоре появились и другие приложения в разных областях математики. Например, градиентные векторные поля замкнутых 1-форм приводят к замечательным результатам в теории динамических систем, и некоторые темы, возникшие на этом пути, изложены в недавней монографии [5] и обзорной статье [6].

Другая удивительная связь была установлена с геометрической теорией групп, когда выяснилось, что работа Ж.-К. Сикорава [7], применившего теорию Новикова к симплектической топологии, находится в тесной связи с работами Р. Бьери, В. Д. Неймана, Р. Штребеля и Б. Ренца [8], [9]. Более подробно геометрические инварианты $\Sigma^{k}(G)$ группы $G$ (их определение мы напомним в разделе 1), содержащие важную информацию о свойствах конечности для некоторых подгрупп $G$, можно описать в терминах обнуления в обобщенных гомологиях Новикова.

Свойство, объединяющее эти области, - существование 1-формы из классов когомологий $\xi \in H^{1}(X ; \mathbb{R})$. Если $X$ - гладкое многообразие, это частный случай теории де Рама, и первым автором настоящей статьи была развита теория 1-форм на топологических пространствах, для которых имеет место более общий результат (см. [10]). Если $X$ связно, то группу $H^{1}(X ; \mathbb{R})$ можно отождествить с множеством гомоморфизмов $\operatorname{Hom}\left(\pi_{1}(X), \mathbb{R}\right)$, где $\mathbb{R}$ рассматривается как группа относительно обычного сложения. Действительно, на инварианты $\Sigma^{k}(G)$ можно смотреть как на подмножества единичной сферы в $\operatorname{Hom}(G, \mathbb{R})$.

Одной из целей данной статьи является описание этих связей в общем случае и развитие теории Бьери-Неймана-Штребеля-Ренца с использованием языка топологии замкнутых 1-форм. В частности, мы обобщаем понятие сигма-инвариантов на случай конечных клеточных комплексов. Эти инварианты $\Sigma^{k}(X)$ для $k \geqslant 1$ определены как обобщения теоретико-групповых версий и имеют аналогичные свойства. Это обобщение мотивировано регулярным появлением теоретико-групповых инвариантов в топологии замкнутых 1-форм. Например, условие $\xi \in \Sigma^{2}\left(\pi_{1}(M)\right)$ возникает в работе $\Phi$. Латура [11] как необходимое для существования невырожденной замкнутой 1-формы в классе когомологий $\xi \in H^{1}(M ; \mathbb{R})$, где $M$-замкнутое многомерное многообразие $(\operatorname{dim} M \geqslant 6)$. Другим необходимым условием в [11], из которого на самом деле следует условие $\xi \in \Sigma^{2}\left(\pi_{1}(M)\right)$, является стягиваемость некоторого функционального пространства. Оказывается, что стягиваемость этого пространства эквивалентна выполнению условия $\xi \in \Sigma^{k}(M)$ для всех $k \geqslant 1$. Заметим, что для трехмерных компактных многообразий условие $\xi \in \Sigma^{1}\left(\pi_{1}(M)\right)$ является достаточным для существования невырожденной замкнутой 1-формы, представляющей $\xi$ (cм. [8]).

Другим свойством этих новых сигма-инвариантов является то, что они отражают свойства конечности для бесконечнолистных абелевых накрытий $q$ : $\bar{X} \rightarrow X$. Под абелевым накрытием мы понимаем регулярное накрытие с абеле- 
вой группой $\pi_{1}(X) / \pi_{1}(\bar{X})$. Обозначим

$$
S(X, \bar{X})=\left\{0 \neq \xi: \pi_{1}(X) \rightarrow \mathbb{R}|\xi|_{\pi_{1}(\bar{X})}=0\right\} .
$$

ТеОРемА. Пусть $X$ - конечный связный клеточный комплекс, и пусть $q: \bar{X} \rightarrow X$ - регулярное накрытие, для которого группа $\pi_{1}(X) / \pi_{1}(\bar{X})$ абелева. При $k \geqslant 1$ следующие свойства эквивалентны:

1) пространство $\bar{X}$ гомотопически эквивалентно клеточному комплексу с конечным $k$-остовом;

2) $S(X, \bar{X}) \subset \Sigma^{k}(X)$.

Более того, если $S(X, \bar{X}) \subset \Sigma^{\operatorname{dim} X}(X)$, то $\bar{X}$ является конечно доминируемым.

Доказательство этой теоремы приводится в разделе 7.

Общим в этих определениях и технике является понятие подвижности подмножеств в пространстве $X$. Здесь подвижность понимается относительно замкнутой 1-формы $\omega$, представляющей класс когомологий $\xi \in H^{1}(X ; \mathbb{R})$. Грубо говоря, подвижность множества $A \subset X$ означает существование гомотопии $H$ множества $A$ в $X$, начинающейся с вложения и такой, что для каждой точки $a \in A$ интеграл от $\omega$ вдоль пути $t \mapsto H_{t}(a)$ является большим. Тогда как гомологические версии, использующие цепные гомотопии, возникли уже в работе [9; теорема C], топологическая версия была сформулирована в совершенно ином контексте при развитии теории Люстерника-Шнирельмана для замкнутых 1-форм (см. [5], [6], [10], [12], [13]). По аналогии ожидаемы похожие результаты, которые мы получим в разделе 4. Понятие подвижности гомологических классов изучается в разделе 8 и имеет приложения при оценке когомологической длины в теории Люстерника-Шнирельмана для замкнутых 1-форм.

Представленная статья написана как сопровождающая недавнего обзора [6], который был посвящен приложениям замкнутых 1-форм к динамическим системам. Она содержит значительное количество нового материала, хотя некоторые части изложены как обзорные.

\section{1. Инварианты Бьери-Неймана-Штребеля-Ренца}

Пусть $G$ - конечно порожденная группа. Напомним определение инвариантов Бьери-Неймана-Штребеля-Ренца $\Sigma^{k}(G ; \mathbb{Z})$, введенных в [8], [9]. Обозначим

$$
S(G)=(\operatorname{Hom}(G, \mathbb{R})-\{0\}) / \mathbb{R}_{+},
$$

т. е. мы отождествляем ненулевые гомоморфизмы, если один из них получается из другого домножением на положительное число. Это пространство является $(r-1)$-мерной сферой, где $r$ - ранг абелизации группы $G$. Мы отождествим $S(G)$ с единичной сферой в пространстве $\operatorname{Hom}(G, \mathbb{R}$ ) (после выбора скалярного произведения в последнем) и будем использовать краткую запись $\xi \in S(G)$.

Для заданного $\xi \in S(G)$ определим подкольцо $\mathbb{Z} G_{\xi}$ кольца $\mathbb{Z} G$ формулой

$$
\mathbb{Z} G_{\xi}=\left\{\sum_{g \in G} n_{g} g \in \mathbb{Z} G \mid n_{g}=0, \text { если } \xi(g)<0\right\} \text {. }
$$


Будем говорить, что тривиальный $\mathbb{Z} G$-модуль $\mathbb{Z}$ имеет тип $F P_{k}$ над $\mathbb{Z} G_{\xi}$, если существует резольвента

$$
\cdots \longrightarrow F_{i} \longrightarrow F_{i-1} \longrightarrow \cdots \longrightarrow F_{0} \longrightarrow \mathbb{Z} \longrightarrow 0
$$

$\mathbb{Z} G$-модуля $\mathbb{Z}$ в свободные $\mathbb{Z} G_{\xi}$-модули, где каждый $F_{i}$ конечно порожден при $i \leqslant k$.

ОПРЕДЕЛЕНИЕ 1. Инварианты Бъери-Неймана-Штребеля-Рениа определяются следующим образом:

$$
\Sigma^{k}(G ; \mathbb{Z})=\left\{\xi \in S(G) \mid \mathbb{Z} \text { имеет тип } F P_{k} \text { над } \mathbb{Z} G_{\xi}\right\} .
$$

Достоинство этих инвариантов заключается в том, что они тесно связаны со свойствами конечности подгрупп группы $G$. Напомним, как формулируются соответствующие свойства конечности.

ОПРЕДЕлЕНиЕ 2. Группа $G$ имеет тип $F P_{k}, k \geqslant 1$, если существует резольвента (1) $\mathbb{Z} G$-модуля $\mathbb{Z}$, где каждый член $F_{i}, i \leqslant k$, является конечно порожденным $\mathbb{Z} G$-модулем. Мы будем говорить, что $G$ имеет тип $F_{k}$, если для $G$ существует пространство Эйленберга-Маклейна с конечным $n$-остовом.

Мы получаем, что если $G$ имеет тип $F_{k}$, то она имеет тип $F P_{k}$ (для этого надо рассмотреть цепной комплекс универсального накрытия пространства Эйленберга-Маклейна), и тип $F P_{1}$ эквивалентен типу $F_{1}$, который означает конечную порожденность. Но из типа $F P_{2}$ не следует конечная представимость, как показывает пример М. Бествины и Н. Бреди [14]. Более подробную информацию о свойствах конечности можно найти в [15], [16].

Заметим, что $\mathbb{Z} G$, рассмотренный как $\mathbb{Z} G$-модуль для любого $\xi$, является прямым пределом свободных $\mathbb{Z} G$-модулей. Следовательно, он является плоским $\mathbb{Z} G_{\xi}$-модулем. Более того, для любого $\mathbb{Z} G$-модуля $A$ имеем $\mathbb{Z} G \otimes_{\mathbb{Z} G_{\xi}} A \cong A$. Следовательно, если $\Sigma^{k}(G ; \mathbb{Z}) \neq \varnothing$ для некоторого $k \geqslant 1$, то можно применить $\mathbb{Z} G \otimes_{\mathbb{Z} G_{\xi}}$ к резольвенте (1) для некоторого $\xi \in \Sigma^{k}(G ; \mathbb{Z})$ - чтобы доказать, что группа $G$ сама имеет тип $F P_{k}$.

Следующая теорема, которую мы обобщим в разделе 7 , связывает свойства конечности некоторых подгрупп с инвариантами.

Теорема 1 (Бьери-Ренц, [9]). Пусть групnа $G$ является группой тиnа $F P_{k}$, a $N$ - подгруппа $G$, содержащая коммутатор группы $G$. Тогда $N$ является группой типа $F P_{k}$ в том и только том случае, когда $\Sigma^{k}(G ; \mathbb{Z})$ содержит подсферу $S(G, N)=\{\xi \in S(G) \mid N \leqslant \operatorname{Ker} \xi\}$.

Имеет место также аналог теоремы 1 , дающий критерий того, когда $N$ является группой типа $F_{k}$, и задействующий гомотопический инвариант $\Sigma^{k}(G)$. Ниже мы опишем другие свойства этого инварианта. Другой результат, доказанный в работе [9], утверждает, что все $\Sigma^{k}(G ; \mathbb{Z})$ являются открытыми множествами в $S(G)$.

В [8] показано, что даже частный случай $k=1$ имеет очень важные приложения в теории групп. 
ТеОрема 2 (Бьери-Нейман-Штребель, [8]). Пусть G - конечно представимая группа, не содержащая свободных неабелевых подгрупп. Тогда

$$
\Sigma^{1}(G ; \mathbb{Z}) \cup-\Sigma^{1}(G ; \mathbb{Z})=S(G) .
$$

Здесь $-\Sigma^{1}(G)$ обозначает образ $\Sigma^{1}(G)$ при антиподальном отображении.

Мы хотим дать более геометрическую интерпретацию этих инвариантов. Временно мы ограничимся случаем $k=1$ и для простоты предположим, что $G$ конечно представима. Пусть $X$ - конечный клеточный комплекс с $\pi_{1}(X) \cong G$, и пусть $q: \bar{X} \rightarrow X-$ универсальное абелево накрытие. Имея ненулевой гомоморфизм $\xi: G \rightarrow \mathbb{R}$, можно построить - индукцией по остовам $\bar{X}$ - отображение $h: \bar{X} \rightarrow \mathbb{R}$ с $h(g x)=\xi(g)+h(x)$ для всех $g \in G$ и $x \in \bar{X}$. Заметим, что $G$ действует на $\bar{X}$ накрывающими преобразованиями и коммутатор действует тривиально. Положим $N=h^{-1}([0, \infty))$, тогда $N$ не обязано быть связным, однако имеет единственную компоненту, на которой $h$ неограничено (см. [8; лемма 5.2]). В следующей теореме мы предполагаем, что отмеченная точка пространства $N$ выбрана в такой компоненте связности.

Теорема 3 (Бьери-Нейман-Штребель, [8]). Включение $\xi \in \Sigma^{1}(G ; \mathbb{Z})$ имеет место тогда и толъко тогда, когда $i_{\#}: \pi_{1}(N) \rightarrow \pi_{1}(\bar{X})$, где $i: N \rightarrow \bar{X}-$ включение, является эпиморфизмом.

Этот геометрический критерий тесно связан с концепцией подвижности подмножества в $X$ относительно данного класса $\xi$ и недавно изучался в связи с теорией Люстерника-Шнирельмана для $\xi$ (см. [10], [5], [6]). Напомним определение замкнутой 1-формы для топологического пространства $X$.

ОПРЕДЕЛЕНИЕ 3. Непрерывная замкнутая 1-борма $\omega$ на топологическом пространстве $X$ определятся как набор $\left\{f_{U}\right\}_{U \in \mathscr{U}}$ непрерывных действительнозначных функций $f_{U}: U \rightarrow \mathbb{R}$, где $\mathscr{U}=\{U\}$ - открытое покрытие пространства $X$ такое, что для любой пары $U, V \in \mathscr{U}$ разность

$$
\left.f_{U}\right|_{U \cap V}-\left.f_{V}\right|_{U \cap V}: U \cap V \rightarrow \mathbb{R}
$$

является локально-постоянной функцией. Другой такой набор $\left\{g_{V}\right\}_{V \in \mathscr{V}}$ (где $\mathscr{V}$ - другое открытое покрытие $X$ ) задает эквивалентную замкнутую 1-форму, если объединенный набор $\left\{f_{U}, g_{V}\right\}_{U \in \mathscr{U}, V \in \mathscr{V}}$ является замкнутой 1-формой, т. е. если для любых $U \in \mathscr{U}$ и $V \in \mathscr{V}$ функция $f_{U}-g_{V}$ является локально-постоянной на пересечении $U \cap V$.

Эти замкнутые 1-формы ведут себя так же, как и гладкие замкнутые 1-формы на многообразиях; их можно интегрировать вдоль путей $\gamma:[a, b] \rightarrow X$ и интегрирование вдоль замкнутых путей определяет гомоморфизм $\xi_{\omega}: \pi_{1}(X) \rightarrow \mathbb{R}$. Более того, любой такой гомоморфизм можно реализовать с помощью замкнутой 1-формы. Подробности см. в [6; §3].

Пример 1. Непрерывная функция $f: X \rightarrow S^{1}$ определяет замкнутую 1-форму следующим образом. Представим окружность $S^{1}$ как факторгруппу $\mathbb{R} / \mathbb{Z}$, и пусть $p: \mathbb{R} \rightarrow \mathbb{R} / \mathbb{Z}$ - проекция. Пусть $I=(a, b) \subset \mathbb{R}-$ открытый интервал и $b-a \leqslant 1$. Тогда $I$ гомеоморфен открытому множеству $p(I) \subset \mathbb{R} / \mathbb{Z}$ с помощью $p$. Набор

$$
\omega=\left\{\left.\left(\left.p\right|_{I}\right)^{-1} \circ f\right|_{f^{-1}(p(I))}: f^{-1}(p(I)) \rightarrow I\right\}
$$


определяет замкнутую 1-форму. Более того, $\xi_{\omega}: \pi_{1}(X) \rightarrow \mathbb{R}$ можно отождествить с $f_{\#}: \pi_{1}(X) \rightarrow \pi_{1}\left(S^{1}\right)$, если стандартная образующая группы $\pi_{1}\left(S^{1}\right)$ отождествлена с $1 \in \mathbb{R}$.

ОПРЕДЕЛЕНИЕ 4. Пусть $X$ - конечный связный клеточный комплекс, $G=$ $\pi_{1}(X)$ и $\omega$ - замкнутая 1-форма на $X$. Подмножество $A \subset X$ называют $n$-noдвижным относительно $\omega$ и отклонения $C \geqslant 0$ (где $n$ - целое число), если найдется такая гомотопия $H: A \times[0,1] \rightarrow X$, что $H(a, 0)=a$ для всех $a \in A$ и

$$
\int_{a}^{H_{1}(a)} \omega \geqslant n, \quad \int_{a}^{H_{t}(a)} \omega \leqslant-C
$$

для всех $a \in A$ и $t \in[0,1]$. Здесь интеграл берется вдоль пути $s \mapsto H(a, s)$ для $s \in[0, t]$.

Понятие подвижности уходит корнями в теорию Люстерника-Шнирельмана для замкнутых 1-форм (ср. [6], [10]). Следующее утверждение показывает, что это понятие дает критерий для $\Sigma^{1}(G ; \mathbb{Z})$.

ПРЕДЛОЖЕНИЕ 1. Пусть $X$ - конечный связный клеточный комплекс, $G=$ $\pi_{1}(X)$ и $\xi: G \rightarrow \mathbb{R}$ - гомоморфизм, представленный замкнутой 1-формой $\omega$. Тогда следующие условия эквивалентнъ:

1) $\xi \in \Sigma^{1}(G ; \mathbb{Z})$

2) существует такое $C \geqslant 0$, что 1 -остов $X^{(1)} \subset X$ является $n$-подвижным относительно $\omega$ и отклонения $C$ для любого $n>0$.

ДоказАтельство. 1$) \Longrightarrow 2$ ). Пусть $\bar{X}$ - универсальное абелево накрытие $X$ и $h: \bar{X} \rightarrow \mathbb{R}-$ прообраз $\omega$ на $\bar{X}$. Легко видеть, что $h(g x)=\xi(g)+h(x)$ для всех $x \in \bar{X}$ и $g \in G$. Выберем $N=h^{-1}([0, \infty))$, и пусть $N^{\prime} \subset N-$ компонента, на которой $h$ неограничено согласно [8; лемма 5.2]. Для любой клетки $\sigma$ пространства $X$ рассмотрим поднятие $\bar{\sigma} \subset h^{-1}((-\infty,-1])$. Если $\sigma$ - нульмерная клетка, то можно определить такое клеточное отображение $H_{\sigma}:[0,1] \rightarrow \bar{X}$, что $H_{\sigma}(0)=\bar{\sigma}$ и $H_{\sigma}(1) \in N^{\prime}$. Здесь $[0,1]$ рассматривается как стандартный клеточный комплекс с двумя нульмерными и одной одномерной клеткой. Используя эквивариантность, получаем эквивариантную клеточную гомотопию $H^{0}: \bar{X}^{(0)} \times[0,1] \rightarrow \bar{X}$. Заметим, что $G$ действует на $\bar{X} \times[0,1]$ по формуле $g(x, t)=(g x, t)$ и $H^{0}$ индуцирует гомотопию на $X^{(0)}$, которая задает 1-подвижность нульмерного остова. Так как образ $H_{1}^{0}$ лежит в нульмерном остове, мы можем итерировать гомотопию для получения $n$-подвижности для любого $n>0$.

Для любой одномерной клетки $\sigma$ пространства $X$ возьмем клетку $\bar{\sigma} \subset$ $h^{-1}((-\infty,-1])$, и пусть $u, v-$ граничные точки $\bar{\sigma}$. Итерируя в случае необходимости $H^{0}$, мы можем считать, что $H^{0}(u, 0), H^{0}(u, 1) \in N^{\prime}$, и поскольку $N^{\prime}$ связно, можно найти клеточное отображение $H_{u, v}:[0,1] \rightarrow N^{\prime}$, соединяющее эти точки.

Заметим, что $H_{u, v}([0,1]), H^{0}(\{u, v\} \times[0,1])$ и $\bar{\sigma}$, будучи подходящим образом ориентированы, образуют замкнутую петлю в $\widetilde{X}$. Но по теореме 3 эта петля представима петлей из $N^{\prime}$. Другими словами, изменяя кривую $H_{u, v}$ в $N^{\prime}$ подходящим образом, можно считать, что эта петля ограничивает. Следовательно, $H^{0}$ можно продолжить до такого клеточного отображения $H_{\sigma}:\left(\bar{X}^{(0)} \cup \bar{\sigma}\right) \times$ 
$[0,1] \rightarrow \bar{X}$, что $H_{\sigma}(\bar{\sigma}, 1)=H_{u, v}([0,1])$. Проделав это со всеми одномерными клетками пространства $X$ и продолжая эквивариантно, получаем клеточную и эквивариантную гомотопию $H^{1}: \bar{X}^{(1)} \times[0,1] \rightarrow \bar{X}$ такую, что $H_{0}^{1}$ является вложением и $h\left(H^{1}(x, 1)\right)-h(x) \geqslant 1$.

Поскольку $H^{1}$ эквивариантно, получаем гомотопию $H: X^{(1)} \times[0,1] \rightarrow X$, которая показывает, что $X^{(1)}$ является 1-подвижным относительно $\omega$. Ввиду компактности существует такое $C>0$, что $X^{(1)}$ является 1-подвижным относительно отклонения $C$. Образ $H_{1}$ снова является 1-остовом пространства $X$, следовательно, мы можем итерировать гомотопию. Заметим, что итерирование гомотопии не увеличивает $C$, поэтому $X^{(1)}$ является $n$-подвижным относительно $\omega$ и $C$ для любого $n>0$.

2) $\Longrightarrow 1)$ Пусть $h: \bar{X} \rightarrow \mathbb{R}$ и $N^{\prime} \subset N=h^{-1}([0, \infty))$ такие как выше. Возьмем такую отмеченную точку $x_{0} \in N^{\prime}$, что $f\left(x_{0}\right) \geqslant C+1$. Пусть $\gamma:\left(S^{1}, 1\right) \rightarrow$ $\left(\widetilde{X}, x_{0}\right)$ - петля, которую можно рассматривать как клеточную. Ввиду компактности $S^{1}$ существует такое $K \leqslant 0$, что $\gamma\left(S^{1}\right) \subset f^{-1}([K, \infty))$.

По предположению существует такая гомотопия $\bar{H}: \bar{X}^{(1)} \times[0,1] \rightarrow \bar{X}$, что $\bar{H}_{0}$ является вложением и

$$
h\left(\bar{H}_{1}(x)\right)-h(x) \geqslant C-K, \quad h\left(\bar{H}_{t}(x)\right)-h(x) \geqslant-C
$$

для всех $x \in \bar{X}$. Пусть $\mu: \widetilde{X} \rightarrow[0,1]$ - такое отображение, что $\left.\mu\right|_{f^{-1}([C+1, \infty))} \equiv 0$ и $\left.\mu\right|_{f^{-1}((-\infty, C])} \equiv 1$. Определим $A: S^{1} \times[0,1] \rightarrow \widetilde{X}$ формулой $A(x, t)=\bar{H}(\gamma(x)$, $\mu(\gamma(x)) \cdot t)$. Тогда $A(x, 0)=\gamma(x), A(x, 1)=\bar{H}(\gamma(x), \mu(\gamma(x))) \in N^{\prime}$ и $A\left(x_{0}, t\right)=x_{0}$ для всех $t \in[0,1]$. Значит, $\xi \in \Sigma^{1}(G ; \mathbb{Z})$ по теореме 3. Предложение 1 доказано.

Критерий, аналогичный условию 2) из предложения 1, приводит к гомотопической версии инвариантов Бьери-Неймана-Штребеля-Ренца $\Sigma^{k}(G)$, введенных в [9]. Предположим, что $X$ - пространство типа Эйленберга-Маклейна с конечным $n$-остовом для некоторого $n \geqslant 1$.

ОПРеДЕЛЕНИЕ 5. Пусть $X$ такое же, как выше, $\xi \in S(G), \omega$ - замкнутая 1 -форма на $X$, представляющая $\xi$, и $k \geqslant 0$. Будем говорить, что $\xi \in \Sigma^{k}(G)$, если существуют $\varepsilon>0$ и клеточная гомотопия $H: X^{(k)} \times[0,1] \rightarrow X$ такие, что $H(x, 0)=x$ для всех $x \in X^{(k)}$ и

$$
\int_{\gamma_{x}} \omega \geqslant \varepsilon
$$

для всех $x \in X^{(k)}$, где $\gamma_{x}:[0,1] \rightarrow X$ задается формулой $\gamma_{x}(t)=H(x, t)$. Здесь $X^{(k)}$ обозначает $k$-остов $X$.

Условие клеточности $H$ гарантирует, что образ $H_{1}$ лежит в $X^{(k)}$ и, следовательно, гомотопию можно итерировать. Поэтому $\varepsilon$ может быть сколь угодно большим, что показывает независимость определения от выбора $\omega$. Все итерации имеют одно и то же отклонения $C \geqslant 0$. Используя клеточную аппроксимацию, легко показать, что условие 2) из предложения 1 эквивалентно условию $\xi \in \Sigma^{1}(G)$.

Вышеприведенное определение не является обычным определением $\Sigma^{k}(G)$, но, как следует из предложения 2 ниже, оно согласуется с определением, данным Р. Бьери и Б. Ренцем [9; §6]. 
Несмотря на то, что в общем случае описать $\Sigma^{k}(G)$ и $\Sigma^{k}(G ; \mathbb{Z})$ достаточно сложно, существуют важные классы групп, для которых сигма-инварианты могут быть определены. Например, это прямоугольные группы Артина (см. [17]) и группа Томсона $F$ (см. [18]). Более подробную информацию об этих инвариантах и их приложениях см., например, в [8], [9], [19]-[21].

\section{2. Сигма-инварианты клеточных комплексов}

Ограничиваться в определении 5 асферическими пространствами $X$ не имеет особого смысла, поэтому мы распространим это определение на более общие $X$. Для простоты предположим, что $X$ - конечный связный клеточный комплекс, но можно рассмотреть случай, когда $X$ имеет конечный $n$-остов для некоторого $n \geqslant 1$ (в этом случае можно определить $\Sigma^{k}(X)$ для $k \leqslant n$ ). Для начала определим

$$
S(X)=\left(\operatorname{Hom}\left(H_{1}(X), \mathbb{R}\right)-\{0\}\right) / \mathbb{R}_{+},
$$

т. е. мы рассматриваем гомоморфизмы с точностью до положительных множителей. Очевидно, что $S(X)=S(G)$, где $G=\pi_{1}(X)$.

Теперь можно дать определение сигма-инвариантов для клеточных комплексов, аналогичное определению 5.

ОПредЕЛЕНИЕ 6 . Пусть $X$ - конечный связный клеточный комплекс, $\xi \in$ $S(X), \omega-$ замкнутая 1-форма на $X$, представляющая $\xi$, и $k \geqslant 0$. Будем говорить, что $\xi \in \Sigma^{k}(X)$, если существуют $\varepsilon>0$ и клеточная гомотопия $H: X^{(k)} \times[0,1] \rightarrow X$ такие, что $H(x, 0)=x$ для всех $x \in X^{(k)}$ и

$$
\int_{\gamma_{x}} \omega \geqslant \varepsilon
$$

для всех $x \in X^{(k)}$, где $\gamma_{x}:[0,1] \rightarrow X$ задается формулой $\gamma_{x}(t)=H(x, t)$.

Пусть $p: \widetilde{X} \rightarrow X$ - универсальное накрытие. Так же как и для гладких многообразий, замкнутая 1-форма $\omega$ поднимается в точную форму на $\widetilde{X}: p^{*} \omega=$ $d h$ для некоторой функции $h: \widetilde{X} \rightarrow \mathbb{R}$ такой, что

$$
h(g x)=h(x)+\xi(g)
$$

для всех $g \in \pi_{1}(X)$ и $x \in \widetilde{X}$. Функция $h: \widetilde{X} \rightarrow \mathbb{R}$ со свойством (2) называется функцией высоты. Такая функция высоты определяет замкнутую 1-форму, представляющую класс $\xi$. Подмножество $N \subset \widetilde{X}$ называется окрестностъю бесконечности относителъно $\xi$, если существуют такие функция высоты $h_{\xi}: \widetilde{X} \rightarrow \mathbb{R}$ и число $a \in \mathbb{R}$, что выполнено

$$
h_{\xi}^{-1}([a, \infty)) \subset N .
$$

Легко проверить, что если $N$ - окрестность бесконечности относительно $\xi$ для некоторой функции высоты, то $N$ также является окрестностью бесконечности относительно $\xi$ для любой другой функции высоты.

Если задана конкретная функция высоты $h_{\xi}$, обозначим $N_{i}=h_{\xi}^{-1}([i, \infty))$ для любого $i \in \mathbb{R}$.

Можно описать $\Sigma^{k}(X)$ в терминах только функции высоты. Для этого нам понадобится еще одно определение. 
ОПРЕДЕЛЕНИЕ 7 . Пусть $X$ - конечный связный клеточный комплекс, $h_{\xi}$ : $\widetilde{X} \rightarrow \mathbb{R}-$ функция высоты и $\xi \in H^{1}(X ; \mathbb{R})$ - ненулевой класс. Путем в бесконечность относительно $\xi$ назовем такое отображение $\gamma:[0, \infty) \rightarrow \widetilde{X}$, что для любой окрестности $N$ бесконечности существует такое $T \geqslant 0$, что $\gamma(t) \in N$ для Bcex $t \geqslant T$.

Если задан путь $\gamma$ к бесконечности, то для любой окрестности $N$ бесконечности можно выбрать точку $\gamma\left(T_{N}\right) \in N$ и получить обратную систему $\left\{\pi_{*}\left(\widetilde{X}, N, \gamma\left(T_{N}\right)\right)\right\}$, где изменение отмеченных точек задается с помощью $\left.\gamma\right|_{\left[T_{N}, T_{N^{\prime}}\right]}$. Мы часто будем опускать в обозначениях отмеченные точки, но хотим обратить внимание, что путь в бесконечность всегда предполагается зафиксированным. Следующее предложение показывает, что отмеченные пути не являются важными для наших целей.

ПРеДЛОЖЕНИЕ 2. Пусть $X-$ конечный связный клеточный комплекс, $\xi \in$ $H^{1}(X ; \mathbb{R})$ - ненулевой класс и $h_{\xi}: \widetilde{X} \rightarrow \mathbb{R}-$ функция высоты. Следующие условия эквивалентны:

1) $\xi \in \Sigma^{k}(X)$;

2) существует такое $\lambda \geqslant 0$, что $j_{\#}: \pi_{l}\left(\tilde{X}, N_{i}\right) \rightarrow \pi_{l}\left(\tilde{X}, N_{i-\lambda}\right)$ является тривиальным для всех $l \leqslant k$ u $i \in \mathbb{R}$;

3) для любой окрестности $N$ бесконечности относительно $\xi$ существует другая окрестность $N^{\prime} \subset N$ такая, что $j_{\#}: \pi_{l}\left(\widetilde{X}, N^{\prime}\right) \rightarrow \pi_{l}(\widetilde{X}, N)$ является тривиальным для всех $l \leqslant k$;

4) существуют $\varepsilon>0$ и эквивариантная клеточная гомотопия $\widetilde{H}: \widetilde{X}^{(k)} \times$ $[0,1] \rightarrow \widetilde{X}$ такие, что $\widetilde{H}_{0}$ является вложением $и h_{\xi}\left(\widetilde{H}_{1}(\tilde{x})\right)-h_{\xi}(\tilde{x}) \geqslant \varepsilon$ для всех $\tilde{x} \in \tilde{X}^{(k)}$.

ДоказАТЕЛЬСтво. 1) $\Longleftrightarrow 4$ ), так как можно поднять $H$ на $\widetilde{H}$ и $\widetilde{H}$ определяет $H$.

Импликация 2) $\Longrightarrow 3$ ) очевидна.

$3) \Longrightarrow 4)$. Определим $H$ по индукции относительно остовов $\widetilde{X}$. Гомотопию всегда можно определить на $\widetilde{X}^{(0)}$, поскольку $\widetilde{X}$ связно и $\xi$ - ненулевой класс. Предположим, что для $H: \widetilde{X}^{(k-1)} \times[0,1] \rightarrow \widetilde{X}$ выполнено условие 4$)$. Пусть $N=N_{0}=h_{\xi}^{-1}([0, \infty))$ - окрестность бесконечности относительно $\xi$, окрестность $N^{\prime} \subset N$ такая же, как в 3$)$, и выберем $\varepsilon>0$. Выберем поднятие $\tilde{\sigma} \subset \tilde{X}-N_{-\varepsilon}$ для любой $k$-мерной клетки $\sigma$ пространства $X$. Итерируя $H$ при необходимости, можно считать, что $H_{1}(\partial \tilde{\sigma}) \subset N^{\prime}$. Имея характеристическую функцию $\chi_{\sigma}:\left(D^{k}, S^{k-1}\right) \rightarrow\left(\widetilde{X}^{(k)}, \widetilde{X}^{(k-1)}\right)$, можно взять композицию $\left.\chi_{\sigma}\right|_{S^{k-1}}$ с $H$, чтобы получить элемент из $\pi_{k}\left(\widetilde{X}, N^{\prime}\right)$, ограничение которого тривиально на $\pi_{k}(\widetilde{X}, N)$. Это дает гомотопию $\chi: D^{k} \times[0,1] \rightarrow \widetilde{X}$ с $\chi_{0}=\chi_{\sigma}$ и $\chi_{1}\left(D^{k}\right) \subset N$. Мы можем использовать эту гомотопию, чтобы продолжить $H$ эквивариантно на $k$-остов так, чтобы выполнялось 4).

Чтобы доказать импликацию 4$) \Longrightarrow 2$ ), заметим, что $H$ можно использовать, чтобы прогомотопировать любое отображение $\varphi:\left(D^{k}, S^{k-1}\right) \rightarrow\left(\widetilde{X}, N_{i}\right)$ в отображение, образ которого лежит в произвольной окрестности бесконечности. Так как отмеченную точку нельзя двигать во время гомотопии, мы должны изменить $H$ в “буферной зоне" $N_{i-\lambda}-N_{i}$ так, чтобы точки, попавшие 
в $N_{i}$, не менялись. Тем не менее можно найти такую $N_{i-\lambda}$, что $\varphi$ можно прогомотопировать в отображение $\left(D^{k}, S^{k-1}\right) \rightarrow\left(\widetilde{X}, N_{i-\lambda}\right)$, что дает условие 1$)$. Предложение 2 доказано.

ЗАмЕчАНИЕ 1. Заметим, что в условии 4) предложения 2 число $\varepsilon>0$ можно выбрать сколь угодно большим: поскольку гомотопия клеточная, мы можем просто итерировать ее для увеличения $\varepsilon$.

Наш следующий результат показывает, что сигма-инварианты являются открытыми множествами в $S(X)$. Теоретико-групповую версию этого утверждения см. в [8], [9]. Для доказательства нам понадобится версия отображения Абеля-Якоби. Пусть $q: \bar{X} \rightarrow X$ - универсальное абелево накрытие пространства $X$ и $r=b_{1}(X)$ - первое число Бетти пространства $X$. Тогда $H_{1}(X)$ действует на $\bar{X}$ трансляциями монодромии и на $\mathbb{R}^{r}=H_{1}(X) \otimes \mathbb{R}$ сдвигами.

Существует эквивариантное отображение $A: \bar{X} \rightarrow \mathbb{R}^{r}$, каноническое с точностью до гомотопии, называемое отображением Абеля-Якоби (см. [22; предложение 1]). В другой конструкции, заметим, что мы имеем канонический эпиморфизм $\pi_{1}(X) \rightarrow \mathbb{Z}^{r}$ факторизации по $H_{1}(X)$. Тогда $A$ является поднятием соответствующего классифицирующего отображения $X \rightarrow\left(S^{1}\right)^{r}$.

Теорема 4. Пусть $X-$ конечный свлзный клеточный комплекс. Для любого $k \geqslant 0$ множество $\Sigma^{k}(X)$ открыто $u \Sigma^{n}(X)=\Sigma^{\operatorname{dim} X}(X)$ для $n \geqslant \operatorname{dim} X$.

ДокАЗАТЕЛЬСтво. Пусть $h: \widetilde{X} \rightarrow \mathbb{R}^{r}$ - композиция накрывающего отображения $\bar{p}: \widetilde{X} \rightarrow \bar{X}$ с отображением Абеля-Якоби $A: \bar{X} \rightarrow \mathbb{R}^{r}$. Тогда для заданного $\xi: \pi_{1}(X) \rightarrow \mathbb{R}$ мы получаем функцию высоты

$$
h_{\xi}=l_{\xi} \circ h, \quad \text { где } l_{\xi}: \pi_{1}(X) /\left[\pi_{1}(X), \pi_{1}(X)\right] \otimes \mathbb{R} \rightarrow \mathbb{R}
$$

определяется формулой $l_{\xi}([g] \otimes t)=\xi(g) t$. Пусть теперь $\widetilde{H}-$ гомотопия из предложения 2,4$)$ для класса $\xi$. Определим отображение $\widetilde{K}: S(X) \times \widetilde{X}^{(k)} \rightarrow \mathbb{R}$ формулой

$$
\widetilde{K}\left(\xi^{\prime}, \tilde{x}\right)=h_{\xi^{\prime}}\left(H_{1}(\tilde{x})\right)-h_{\xi^{\prime}}(\tilde{x}) .
$$

Выбором $H$ получим $\widetilde{K}(\xi, \tilde{x}) \geqslant \varepsilon$ для всех $\tilde{x} \in \tilde{X}$ и для некоторого $\varepsilon>0$. Отображение $\widetilde{K}$ также индуцирует отображение $K: S(X) \times X^{(k)} \rightarrow \mathbb{R}$. Из компактности $X^{(k)}$ следует, что $\widetilde{K}\left(\xi^{\prime}, \tilde{x}\right) \geqslant \varepsilon / 2$ для всех $\tilde{x} \in \widetilde{X}^{(k)}$ и всех $\xi^{\prime}$ из некоторой окрестности класса $\xi$ в $S(X)$. Следовательно, условие 4) предложения 2 выполнено для всех таких $\xi^{\prime}$. Теорема 4 доказана.

Получаем следующую связь между $\Sigma^{k}(X)$ и его теоретико-групповой версией $\Sigma^{k}\left(\pi_{1}(X)\right)$.

ПРЕДЛОЖЕНИЕ 3. Пусть $X$ - конечный связный клеточный комплекс $u$ $k \geqslant 0$. Если $\widetilde{X}$ является $k$-связным, то

$$
\Sigma^{k}(X)=\Sigma^{k}\left(\pi_{1}(X)\right) \quad u \quad \Sigma^{k+1}(X) \subset \Sigma^{k+1}\left(\pi_{1}(X)\right) .
$$

Вложение может быть собственными, как показывает пример $X=\mathbb{S}^{1} \vee \mathbb{S}^{k}$ при $k \geqslant 2$. 
ДокАЗАТЕЛЬСтво. Заметим, что $K\left(\pi_{1}(X), 1\right)$ можно сконструировать из пространства $X$ приклейкой $n$-мерных клеток при $n \geqslant k+2$. Обозначим это пространство Эйленберга-Маклейна через $Y$. Если $\xi \in \Sigma^{k+1}(X)$, то клеточная гомотопия $\widetilde{H}: \widetilde{X}^{(k+1)} \times[0,1] \rightarrow \widetilde{X}$ из предложения 2,4$)$ индуцирует гомотопию $\widetilde{H}^{\prime}: \widetilde{Y}^{(k+1)} \times[0,1] \rightarrow \widetilde{Y}$, поскольку $\widetilde{Y}^{(k+1)}=\widetilde{X}^{(k+1)}$ и $\widetilde{X} \subset \widetilde{Y}$. Следовательно, $\xi \in \Sigma^{k+1}\left(\pi_{1}(X)\right)$.

Если $\xi \in \Sigma^{k}\left(\pi_{1}(X)\right)$, то мы имеем клеточную гомотопию $\widetilde{H}: \widetilde{Y}^{(k)} \times[0,1] \rightarrow$ $\tilde{Y}^{(k+1)}$, как и в предложении 2,4$)$, и, поскольку $\widetilde{Y}^{(k+1)}=\widetilde{X}^{(k+1)}$, это доказывает включение $\xi \in \Sigma^{k}(X)$. Предложение 3 доказано.

По аналогии с гомологическими инвариантами $\Sigma^{k}(G, \mathbb{Z})$ Бьери и Ренца [9], мы хотим определить гомологические инварианты $\Sigma^{k}(X, \mathbb{Z})$. На самом деле мы введем более общее определение для цепных комплексов, которое также обобщает инварианты из [9]. Предположим, что все цепные комплексы удовлетворяют условию $C_{i}=0$ при $i<0$.

ОПРЕДЕЛЕНИЕ 8 . Пусть $R$ - кольцо, $n$ - неотрицательное целое число и $C-$ цепной комплекс над $R$. Говорят, что $C$ имеет конечный $n$-mun, если существуют конечно порожденный проективный цепной комплекс $C^{\prime}$ и цепное отображение $f: C^{\prime} \rightarrow C$ такие, что $f_{i}: H_{i}\left(C^{\prime}\right) \rightarrow H_{i}(C)$ являются изоморфизмом при $i<n$ и эпиморфизмом при $i=n$. Будем называть такое $f n$-эквивалентностью.

Очевидно, что это эквивалентно существованию свободного цепного комплекса $D$ над $R$ и цепного отображения $f: D \rightarrow C$, индуцирующего изоморфизм в гомологиях. Дополнительно, $D_{i}$ являются конечно порожденными при $i \leqslant n$.

ОПРЕДЕЛЕНИЕ 9. Пусть $C$ - цепной комплекс над $\mathbb{Z} G$ и $k \geqslant 0$. Тогда

$$
\Sigma^{k}(C)=\left\{\xi \in S(G) \mid C \text { имеет конечный } k \text {-тип над } \mathbb{Z} G_{\xi}\right\} .
$$

ОПРЕДЕЛЕНИЕ 10. Если $X$ - конечный связный цепной комплекс, положим

$$
\Sigma^{k}(X ; \mathbb{Z})=\Sigma^{k}\left(C_{*}(\widetilde{X})\right)
$$

Инварианты $\Sigma^{k}(G ; A)$ Бьери и Ренца [9] задаются равенствами $\Sigma^{k}(G ; A)=$ $\Sigma^{k}(P)$, где $P$ - проективная $\mathbb{Z} G$-резольвента $\mathbb{Z} G$-модуля $A$.

ЗАмЕчАНиЕ 2. Заметим, что $\mathbb{Z} G$ является плоским $\mathbb{Z} G_{\xi}$-модулем, поскольку $\mathbb{Z} G$ - прямой предел свободных $\mathbb{Z} G_{\xi}$-модулей. Так как $\mathbb{Z} G \otimes_{\mathbb{Z} G_{\xi}} A \cong A$ для любого $\mathbb{Z} G$-модуля $A$, из $\xi \in \Sigma^{n}(C)$ следует, что $C$ имеет $n$-тип над $\mathbb{Z} G$. Если цепной комплекс $C$ является свободным, что он цепно-гомотопически эквивалентен комплексу $D$ такому, что модули $D_{i}$ являются конечно порожденными при $i \leqslant n$.

Чтобы получить аналог предложения 2, введем определение функции высоты в случае цепного комплекса.

ОПРЕДЕЛЕНИЕ 11. Пусть $C$ - конечно порожденный свободный цепной комплекс над $\mathbb{Z} G$ и $\xi: G \rightarrow \mathbb{R}$ - ненулевой гомоморфизм. Нормированием на $C$, 
продолюающим $\xi$, называется набор отображений $v: C_{k} \rightarrow \mathbb{R}_{\infty}$, удовлетворяющих следующим условиям:

1) $v(a+b) \geqslant \min \{v(a), v(b)\}$ для всех $a, b \in C_{k}$;

2) $v(g a)=\xi(g)+v(a)$ для всех $g \in G, a \in C_{k}$;

3) $v(-a)=v(a)$ для всех $a \in C_{k}$.

4) $v(\partial a) \geqslant v(a)$ для всех $a \in C_{k}$;

5) $v^{-1}(\{\infty\})=\{0\}$.

Здесь $\mathbb{R}_{\infty}$ обозначает множество действительных чисел с добавленной бесконечностью $\infty$ и очевидным продолжением сложения и сравнения.

Для определения нормирования на свободном $\mathbb{Z} G$-комплексе $C$, являющемся конечно порожденным в каждой размерности, обозначим через $X_{i}$ базис в $C_{i}$. Положим $v(x)=0$ для всех $x \in X_{0}$. Тогда нормирование очевидным образом продолжается на $C_{0}$. По индукции определим нормирование для всех $x \in C_{i}$ следующим образом: $v(x)=0$ при $\partial x=0$ и $v(x)=v(\partial x)$, при $\partial x \neq 0$. И снова $v$ можно продолжить на $C_{i}$, что даст существование нормирований на $C$.

ПРЕДЛОЖЕНИЕ 4. Пусть $X$ - конечный связный клеточный комплекс, $\xi \in H^{1}(X ; \mathbb{R})$ - ненулевой класс и $v: C_{*}(\widetilde{X}) \rightarrow \mathbb{R}_{\infty}-$ нормирование, продолжающее $\xi$. Следующие условия эквивалентны:

1) $\xi \in \Sigma^{k}(X, \mathbb{Z})$

2) существует такое $\lambda \geqslant 0$, что $j_{*}: H_{l}\left(\widetilde{X}, N_{i}\right) \rightarrow H_{l}\left(\widetilde{X}, N_{i-\lambda}\right)$ является тривиальным для всех $l \leqslant k$ и любого $i \in \mathbb{R}$;

3) для любой окрестности $N$ бесконечности относительно $\xi$ существует другая окрестность $N^{\prime} \subset N$ такая, что $j_{*}: H_{l}\left(\widetilde{X}, N^{\prime}\right) \rightarrow H_{l}(\widetilde{X}, N)$ является тривиальным для всех $l \leqslant k$;

4) для заданного $\varepsilon>0$ существует $\mathbb{Z} G$-цепное отображение $A: C_{*}(\widetilde{X}) \rightarrow$ $C_{*}(\tilde{X})$, иепно-гомотопное тождественному, такое, ито с $v(A(x)) \geqslant$ $v(x)+\varepsilon$ для всех ненулевых $x \in C_{i}(\widetilde{X})$ при $i \leqslant k$.

Доказательство эквивалентности условий 2), 3) и 4) аналогично доказательству предложения 2 и поэтому опускается. Что же касается условия 1), мы отсылаем читателя к приложению А, где приведена более общая версия.

СлЕДСТВИЕ 1. Пусть $X$ - конечный связный клеточный комплекс. Для любого $k \geqslant 0$ множество $\Sigma^{k}(X, \mathbb{Z})$ открыто и $\Sigma^{n}(X, \mathbb{Z})=\Sigma^{\operatorname{dim} X}(X, \mathbb{Z})$ при $n \geqslant \operatorname{dim} X$.

СлеДСТвИЕ 2. Пусть $X-$ конечный связный клеточный комплекс. Тогда:

1) $\Sigma^{1}(X)=\Sigma^{1}(X, \mathbb{Z})$;

2) $\Sigma^{k}(X) \subset \Sigma^{k}(X, \mathbb{Z})$ npu $k \geqslant 2$.

ДокАзАтЕльство. Легко видеть, что из утверждение 3) предложения 2 следует условие 3$)$ предложения 4 , следовательно, $\Sigma^{k}(X) \subset \Sigma^{k}(X, \mathbb{Z})$ при $k \geqslant 1$. Кроме того, при $k=1$ клеточную гомотопию из утверждения 4,3$)$ можно использовать для построения гомотопии, как это сделано в доказательстве предложения 2,3$)$.

Как следует из примера работы [14], в общем случае $\Sigma^{2}(X) \neq \Sigma^{2}(X, \mathbb{Z})$ (см. [17]). 


\section{3. Кольцо Новикова и гомологии}

Пусть $G$ - конечно порожденная группа, $\xi \in S(G)$ и

$$
\widehat{\mathbb{Z} G}_{\xi}=\left\{\sum_{g \in G} n_{g} g \mid \#\left\{g \mid n_{g} \neq 0 \text { и } \xi(g)>t\right\}<\infty \text { для всех } t \in \mathbb{R}\right\}
$$

- кольцо, содержащее $\mathbb{Z} G$. Если $\xi: G \rightarrow \mathbb{R}$ инъективно, это так называемое Кольщо Новикова, введенное в [2]. В случае общего $\xi$ мы будем называть это кольцо кольцом Новикова-Сикорава (впервые введено в [7]).

Связь с инвариантами Бьери-Неймана-Штребеля-Ренца обнаружилась сразу, как только стала известна работа Сикорава [7]. Эта связь имеет место и в нашей ситуации и будет объяснена в нижеприведенном предложении 5 .

Если $X$ - конечный связный клеточный комплекс и $p: \bar{X} \rightarrow X-$ регулярное накрытие с $\pi_{1}(\bar{X}) \subset \operatorname{Ker} \xi$, то мы получаем индуцированный гомоморфизм, также обозначаемый через $\xi: G \rightarrow \mathbb{R}$, где $G=\pi_{1}(X) / \pi_{1}(\bar{X})$. Далее мы получаем свободный конечно порожденный $\widehat{\mathbb{Z}}_{\xi}$-цепной комплекс, полагая

$$
C_{*}\left(X ; \widehat{\mathbb{Z} G}_{\xi}\right)=\widehat{\mathbb{Z} G}_{\xi} \otimes_{\mathbb{Z} G} C_{*}(\bar{X}),
$$

мы обозначаем через

$$
H_{*}\left(X ; \widehat{\mathbb{Z} G}_{\xi}\right)=H_{*}\left(C_{*}\left(X ; \widehat{\mathbb{Z} G}_{\xi}\right)\right)
$$

полученные гомологии, которые называются гомологиями Новикова-Сикорава (гомологиями Новикова, если $\xi$ инъективно).

Случай универсального накрытия непосредственно связан с инвариантом $\Sigma^{k}(X)$, но абелево накрытие играет важную роль в оценках категории Люстерника-Шнирельмана.

ПРЕДЛОЖЕНИЕ 5. Пусть $X$ - конечный связный клеточный комплекс, $\xi \in H^{1}(X ; \mathbb{R})$ - ненулевой класс когомологий, $\widetilde{X}$ - универсальное накрытие $X$ u $G=\pi_{1}(X)$. Тогда следующие условия эквивалентны:

1) $\xi \in \Sigma^{k}(X ; \mathbb{Z})$;

2) $H_{i}\left(X ; \widehat{\mathbb{Z} G}_{-\xi}\right)=0$ для $i \leqslant k$.

Заметим, что для проверки включения $\xi \in \Sigma^{k}(X ; \mathbb{Z})$ мы должны использовать кольцо Новикова-Сикорава для $-\xi$. Смысл в этом такой, что мы допускаем бесконечно много ненулевых коэффициентов среди элементов $\widehat{\mathbb{Z}}_{\xi}$ в "отрицательном направлении", что находится в прямом соответствии с оригинальным определением кольца Новикова [1]. Но чтобы придерживаться соглашений из [9], нужно пополнение в “положительную сторону". Чтобы остаться в рамках соглашений, использованных в [6], мы ввели выше знак минус.

ДокАЗАТЕЛЬСТво ПРЕДЛОЖЕНИЯ 5.1$) \Longrightarrow 2)$. Пусть $A: C_{*}(\widetilde{X}) \rightarrow C_{*}(\tilde{X})-$ цепное отображение, цепно-гомотопное тождественному, заданному в предложении 4,4$)$. Тогда id-A: $C_{i}\left(X ; \widehat{\mathbb{Z} G}_{-\xi}\right) \rightarrow C_{i}\left(X ; \widehat{\mathbb{Z}}_{-\xi}\right)$ является изоморфизмом с обратным отображением id $+A+A^{2}+\cdots$, которое сходится $\widehat{\mathbb{Z}}_{-\xi}$ согласно предложению 4,4$)$. Следовательно, отображение в гомологиях одновременно является изоморфизмом и нулевым, поэтому гомологии равны нулю. 
$2) \Longrightarrow 1)$. Поскольку $C_{*}\left(X ; \widehat{\mathbb{Z} G}_{-\xi}\right)$ свободный и ограниченный снизу, зануление гомологических групп до размерности $k$ гарантирует существование цепной гомотопии $\delta: C_{*}\left(X ; \widehat{\mathbb{Z} G}_{-\xi}\right) \rightarrow C_{*+1}\left(X ; \widehat{\mathbb{Z} G}_{-\xi}\right)$ такой, что $\partial_{i+1} \delta_{i}+\delta_{i-1} \partial_{i}=\mathrm{id}$ при $i \leqslant k$. "Отрезание" задает цепную гомотопию $\bar{\delta}: C_{*}(\widetilde{X}) \rightarrow C_{*}(\widetilde{X})$ такую, что $\partial_{i+1} \bar{\delta}_{i}+\bar{\delta}_{i-1} \partial_{i}=\mathrm{id}-A$. Цепное отображение $A$ гомотопно тождественному, и, достаточно хорошо аппроксимируя $\delta$ с помощью $\bar{\delta}$, получаем, что условие 4$)$ из предложения 4 выполнено.

Предложение 5 доказано.

\section{4. Связь с категорией Люстерника-Шнирельмана для замкнутых 1-форм}

Теперь мы хотим описать связь с теорией Люстерника-Шнирельмана замкнутых 1-форм, которая была введена в ряде статей [10], [12], [13]. Более детальную информацию, в частности, приложения и вычисления, можно найти в [5], [6]. Существует множество разных понятий, одно из которых тесно связано с $\Sigma^{k}(X)$ и обозначается $\operatorname{Cat}(X, \xi)$. Здесь $X-$ снова конечный клеточный комплекс и $\xi \in H^{1}(X ; \mathbb{R})$. Напомним определение из [6].

ОПРЕДЕЛЕНИЕ 12. Пусть $X$ - конечный клеточный комплекс, и пусть $\xi \in H^{1}(X ; \mathbb{R})$ - класс когомологий. Зафиксируем замкнутую 1-форму $\omega$, представляющую класс $\xi$. Тогда $\operatorname{Cat}(X, \xi)$ определяется как минимальное целое $k$, для которого существует открытое множество $U \subset X$, удовлетворяющее следующим условиям:

1) $\operatorname{cat}_{X}(X-U) \leqslant k$;

2) для некоторой гомотопии $h: U \times[0, \infty) \rightarrow X$ имеем

$$
h(x, 0)=x \quad \text { и } \quad \lim _{t \rightarrow \infty} \int_{x}^{h_{t}(x)} \omega=-\infty
$$

для любой точки $x \in U$;

3) предел из условия 2) является равномерным по $x \in U$.

Интеграл берется вдоль пути $\gamma:[0, t] \rightarrow X$, заданного равенством $\gamma(\tau)=h(x, \tau)$.

Здесь $\operatorname{cat}_{X}(A)$ для $A \subset X-$ минимальное число $i$, для которого существуют открытые множества $U_{1}, \ldots, U_{i} \subset X$, покрывающие $A$, каждое из которых стягиваемо в $X$. Инвариант $\operatorname{Cat}(X, \xi)$ возник в работе [12]. Легко видеть, что он не зависит от умножения $\xi$ на положительное число и, следовательно, корректно определен на $\xi \in S(X)$. Заметим, что он также определен при $\xi=0$. В последнем случае мы получаем классическую категорию Люстерника-Шнирельмана пространства $X$. Более подробно см. [6].

Связь с $\Sigma^{k}(X)$ описывает следующая теорема. Из-за нашего соглашения о выборе знака мы получим знак минус перед $\xi$.

ТЕОРема 5. Пусть $X$ - конечный связный клеточный комплекс, и пусть $\xi \in H^{1}(X ; \mathbb{R})$ - ненулевой класс когомологий. Положим $n=\operatorname{dim} X$. Если $-\xi \in \Sigma^{k}(X)$ для некоторого $k \leqslant n$, mo $\operatorname{Cat}(X, \xi) \leqslant n-k$. 
ДокАЗАТЕЛЬство. Пусть $U$ - достаточно маленькая открытая окрестность $X^{(k)}$ в $X$, которая ретрагируется на $X^{(k)}$. Пусть $H: X^{(k)} \times[0,1] \rightarrow X-$ гомотопия, начинающиеся с вложения, которое поднимается до гомотопии, как в предложении 2, 3). Определим $h: X^{(k)} \times[0, \infty) \rightarrow X$ равенством $h(x, t)=$ $H\left(H^{m}(x, 1), t-m\right)$, где $m$ - целое число и $t \in[m, m+1]$. Заметим, что $H-$ клеточное отображение, поэтому $H^{m}(x, 1) \in X^{(k)}$. Легко видеть, что комбинация $h$ и ретракции $U$ на $X^{(k)}$ дает требуемую в определении 12 гомотопию. Как хорошо известно, $\operatorname{cat}_{X}\left(X-X^{(k)}\right) \leqslant \operatorname{dim}(X)-k$, откуда и следует результат. Теорема 5 доказана.

Существуют другие определения категории Люстерника-Шнирельмана класса $\xi$, так называемые $\operatorname{cat}(X, \xi)$ и $\operatorname{cat}^{1}(X, \xi)$ (см. [10], [5], [13], [6]), удовлетворяющие неравенствам

$$
\operatorname{cat}(X, \xi) \leqslant \operatorname{cat}^{1}(X, \xi) \leqslant \operatorname{Cat}(X, \xi)
$$

Таким образом, теорема 5 дает верхнюю оценку и для них.

\section{5. Результат типа Гуревича}

Если предположить, что $\xi \in \Sigma^{2}\left(\pi_{1}(X)\right)$, то можно получить обратное утверждение к следствию 2. Это можно описать следующим образом.

ОПРЕДЕЛЕНИЕ 13. Пусть $G$ - конечно представимая группа и $\xi: G \rightarrow \mathbb{R}-$ ненулевой гомоморфизм. Пусть $X$ - конечный связный клеточный комплекс с фундаментальной группой $G$. Будем говорить, что $\xi \in \Sigma^{2}(G)$, если существует такое $\lambda \geqslant 0$, что $j_{\#}: \pi_{l}\left(N_{i}\right) \rightarrow \pi_{l}\left(N_{i-\lambda}\right)$ тривиально для $l \leqslant 1$ и любого $i \in \mathbb{R}$.

Заметим, что можно считать, что $\xi \in H^{1}(X ; \mathbb{R})$, так что окрестности бесконечности определены как выше.

TеОРема 6. Пусть $X$ - конечный связный клеточный комплекс и класс $\xi \in H^{1}(X ; \mathbb{R})$ - ненулевой. Пусть $k \geqslant 2$. Если $\xi \in \Sigma^{2}\left(\pi_{1}(X)\right) \cap \Sigma^{k}(X, \mathbb{Z})$, то $\xi \in \Sigma^{k}(X)$.

Эта теорема вытекает из следующих двух лемм.

ЛЕмма 1. Пусть $X$ - конечный связный клеточный комплекс, и пусть $\xi \in$ $H^{1}(X ; \mathbb{R})$ - ненулевой класс. Если $\xi \in \Sigma^{2}\left(\pi_{1}(X)\right), \operatorname{mo}\left\{\pi_{2}(\widetilde{X}, N)\right\} u\left\{H_{2}(\widetilde{X}, N)\right\}$ являются произоморфными.

ДокАЗАТЕЛЬСтво. Заметим, что

$$
\operatorname{Im}\left(\pi_{2}\left(\tilde{X}, N_{i}\right) \rightarrow \pi_{2}\left(\tilde{X}, N_{i-\lambda}\right)\right)=\operatorname{Im}\left(\pi_{2}(\tilde{X}) \rightarrow \pi_{2}\left(\tilde{X}, N_{i-\lambda}\right)\right),
$$

если любая окружность в $N_{i}$ ограничивает диск в $N_{i-\lambda}$, в частности, образы являются абелевыми. Поскольку $\xi \in \Sigma^{2}\left(\pi_{1}(X)\right)$, можно определить гомоморфизм $\pi_{2}\left(\widetilde{X}, N_{i}\right) \rightarrow H_{2}\left(\widetilde{X}, N_{i-\lambda}\right)$ как в стандартном доказательстве классической теоремы Гуревича (после возможного увеличения $\lambda$ ). См., например, [23]. Детали оставляем читателю. 
ЛЕмма 2. Пусть $X$ - конечный свлзный клеточный комплекс и класс $\xi \in$ $H^{1}(X ; \mathbb{R})$ - ненулевой. Если $k \geqslant 3 u \xi \in \Sigma^{k-1}(X), \operatorname{mo}\left\{\pi_{k}(\widetilde{X}, N)\right\} u\left\{H_{k}(\widetilde{X}, N)\right\}$ произоморфньь.

ДокАЗАТЕльство. Доказательство аналогично доказательству леммы 1, но проще, поскольку мы можем определить гомоморфизм напрямую.

ЗАмечание 3. Теорема 6 также следует из теорем Латура 5.10 и 5.3 в [11].

\section{6. Свойство функториальности}

ПРЕДЛОЖЕНИЕ 6. Пусть $X$ и $Y$ - конечные клеточные комплексы, и пусть $f: X \rightarrow Y$ u $g: Y \rightarrow X-$ такие отображения, что $f g \simeq \operatorname{id}_{Y}$. Тогда для любого $k \geqslant 0$ имеем

$$
\left(f^{*}\right)^{-1}\left(\Sigma^{k}(X)\right) \subset \Sigma^{k}(Y), \quad\left(f^{*}\right)^{-1}\left(\Sigma^{k}(X, \mathbb{Z})\right) \subset \Sigma^{k}(Y, \mathbb{Z}) .
$$

ДокАЗАТЕЛьСтво. Мы дадим доказательство для гомотопического инварианта $\Sigma^{k}(Y)$. Выберем поднятия $\tilde{f}: \widetilde{X} \rightarrow \widetilde{Y}$ и $\tilde{g}: \widetilde{Y} \rightarrow \widetilde{X}$ такие, что $\tilde{f} \tilde{g} \simeq \mathrm{id}_{\tilde{Y}}$ эквивариантно. Пусть $\xi \in S(Y)$ удовлетворяет $f^{*}(\xi) \in \Sigma^{k}(X)$. Пусть $h_{\xi}: \widetilde{Y} \rightarrow \mathbb{R}-$ функция высоты. Тогда $h_{\xi} \circ \tilde{f}: \tilde{X} \rightarrow \mathbb{R}$ является функцией высоты для $f^{*} \xi$.

В силу кокомпактности существует такое $C \geqslant 0$, что $\left|h_{\xi}(\tilde{f} \tilde{g}(\tilde{y}))-h_{\xi}(\tilde{y})\right| \leqslant C$ для любого $\tilde{y} \in \tilde{Y}$.

По предположению, предложению 2 и замечанию 1, существует эквивариантная гомотопия $\widetilde{H}: \widetilde{X}^{(k)} \times[0,1] \rightarrow \widetilde{X}$, начинающаяся с вложения и такая, что $h_{\xi} \tilde{f}(H(\tilde{x}, 1))-h_{\xi} \tilde{f}(\tilde{x}) \geqslant C+1$ для всех $\tilde{x} \in \widetilde{X}$. Следовательно,

$$
h_{\xi} \tilde{f}(H(\tilde{g}(\tilde{y}), 1))-h_{\xi}(\tilde{y}) \geqslant 1 .
$$

Комбинируя $\tilde{f} H(\tilde{g}, \cdot)$ с гомотопией $\tilde{f} \tilde{g} \simeq \mathrm{id}_{\tilde{Y}}$, получаем, что $\xi \in \Sigma^{k}(Y)$. Предложение 6 доказано.

СлеДСтвиЕ 3. Пусть $X$ и $Y$ - конечные связные клеточные комплексы $u h: X \rightarrow Y$ - гомотопическая эквивалентность. Тогда $h^{*}\left(\Sigma^{k}(Y)\right)=\Sigma^{k}(X)$ $u h^{*}\left(\Sigma^{k}(Y, \mathbb{Z})\right)=\Sigma^{k}(X, \mathbb{Z})$ для всех $k \geqslant 0$.

ПРЕДЛОЖЕНИЕ 7. Пусть $X$ и $Y$-конечные клеточные комплексы, и пусть $f: X \rightarrow Y m$-связно для $m \geqslant 1$. Тогда

$$
\left(f^{*}\right)^{-1}\left(\Sigma^{k}(X)\right) \subset \Sigma^{k}(Y), \quad\left(f^{*}\right)^{-1}\left(\Sigma^{k}(X, \mathbb{Z})\right) \subset \Sigma^{k}(Y, \mathbb{Z})
$$

для всех $k \leqslant m$.

ДокАзАТЕЛЬство. Добавим к пространству $X$ клетки в размерностях $\geqslant m+1$, чтобы получить (возможно, бесконечный) клеточный комплекс $X^{\prime}$, содержащий $X$ и такой, что $f$ продолжается до гомотопической эквивалентности $f^{\prime}: X^{\prime} \rightarrow Y$. Пусть $g: Y \rightarrow X^{\prime}$ - обратная клеточная гомотопия. Тогда $g\left(Y^{(m)}\right) \subset X$. Если $f^{*}(\xi) \in \Sigma^{k}(X)$, то гомотопию $H: \widetilde{X}^{(k)} \times[0,1] \rightarrow \widetilde{X}$ можно использовать так же, как и в доказательстве предложения 6 , чтобы показать, что $\xi \in \Sigma^{k}(Y)$ при $k \leqslant m$. Предложение 7 доказано. 
Пример 2. Пусть $X$-конечный связный клеточный комплекс и $f: X \rightarrow X-$ некоторое отображение. Тором этого отображения $M_{f}$ называется факторпространство $M_{f}=X \times[0,1] / \sim$, где $(x, 0) \sim(f(x), 1)$. Существует естественное отображение $g: M_{f} \rightarrow \mathbb{S}^{1}$, заданное равенством $g([x, t])=\exp \{2 \pi i t\}$. Пусть

$$
\xi=[g] \in\left[M_{f}, \mathbb{S}^{1}\right]=H^{1}\left(M_{f} ; \mathbb{Z}\right) \subset H^{1}\left(M_{f} ; \mathbb{R}\right) .
$$

Гомотопия $h: M_{f} \times[0,1] \rightarrow M_{f}$, заданная следующим образом:

$$
H([x, t], s)= \begin{cases}{[x, t-s],} & t \geqslant s, \\ {[f(x), 1+t-s],} & t \leqslant s,\end{cases}
$$

показывает, что $-\xi \in \Sigma^{k}\left(M_{f}\right)$ для всех $k \geqslant 0$.

ПРЕДЛОЖЕНИЕ 8. Пусть $X$ - конечный связный клеточный комплекс $u$ $\xi \in H^{1}(X ; \mathbb{Z})$ - ненулевой класс. Пусть $q: \bar{X} \rightarrow X-$ бесконечное ииклическое накрытие, соответствующее $\operatorname{Ker} \xi$. Предположим, что $\bar{X}$ гомотопически эквивалентно иепному комплексу $Y$ с конечным $k$-остовом. Тогда $\pm \xi \in \Sigma^{k}(X)$.

ДокАЗАтЕЛЬство. Пусть $h: \bar{X} \rightarrow \mathbb{R}$ индуцировано функцией высоты $h_{\xi}$ : $\widetilde{X} \rightarrow \mathbb{R}$ и $\zeta: \bar{X} \rightarrow \bar{X}$ - накрывающая трансляция с $h \zeta(x)>h(x)$ для всех $x \in \bar{X}$. Пусть $a: Y \rightarrow \bar{X}$ и $b: \bar{X} \rightarrow Y$ являются взаимно обратными гомотопическими эквивалентностями. Тогда существует гомотопическая эквивалентность $g: M_{b \zeta a} \rightarrow X$, заданная как

$$
M_{b \zeta a} \simeq M_{\zeta a b} \simeq M_{\zeta} \simeq X
$$

где последняя гомотопическая эквивалентность задана как $[\bar{x}, t] \rightarrow q(\bar{x})$ для $\bar{x} \in \bar{X}$.

Можно предположить, что $b \zeta a: Y \rightarrow Y$ отображает $k$-остов в $k$-остов. Пусть $\varphi: Y^{(k)} \rightarrow Y^{(k)}$ - ограничение $b \zeta a$ на $Y^{(k)}$. Индуцированное отображение $M_{\varphi} \rightarrow M_{b \zeta a}$ является $k$-связным, и поэтому существует $k$-связное отображение $f: M_{\varphi} \rightarrow X$. В силу примера 2 верно включение $-f^{*}(\xi) \in \Sigma^{k}\left(M_{\varphi}\right)$. Из предложения 7 следует, что $-\xi \in \Sigma^{k}(X)$. Для получения $\xi \in \Sigma^{k}(X)$ заменим $\zeta$ на $\zeta^{-1}$. Предложение 8 доказано.

В следующем разделе мы покажем, что имеет место и утверждение, обратное к предложению 8.

\section{7. Результаты доминирования для накрывающих пространств}

Пусть $X$ - конечный связный клеточный комплекс и $q: \bar{X} \rightarrow X-$ регулярное накрытие с абелевой группой монодромии $\pi_{1}(X) / \pi_{1}(\bar{X})$. Тогда мы определяем

$$
S(X, \bar{X})=\left\{\xi \in S(X) \mid q^{*} \xi=0\right\} .
$$

В частности, если $\bar{X}$ универсальное абелево накрытие пространства $X$, то $S(X, \bar{X})=S(X)$. Более общим образом, $S(X, \bar{X})$ является $(d-1)$-мерной сферой, где $d$ - ранг конечно порожденной абелевой группы $\pi_{1}(X) / \pi_{1}(\bar{X})$. 
ТеОрема 7. Пусть $X$ - конечный связный клеточный комплекс, и пусть $q: \bar{X} \rightarrow X$ - регулярное накрытие с абелевой группой монодромии $\pi_{1}(X) / \pi_{1}(\bar{X})$. Если $k \geqslant 1$, то следующие условия эквивалентны:

1) $\bar{X}$ гомотопически эквивалентно пространству с конечным $k$-остовом;

2) $S(X, \bar{X}) \subset \Sigma^{k}(X)$.

Более того, если $S(X, \bar{X}) \subset \Sigma^{\operatorname{dim} X}(X)$, то $\bar{X}$ является конечно доминируемым.

Мы получим теорему 7 из ее более общей версии для цепных комплексов, которая является обобщением теоремы В из [9]. Чтобы получить другое доказательство импликации 1$) \Longrightarrow 2$ ), можно использовать технику из [24; теорема 3.2].

Если $N$ - нормальная подгруппа группы $G$ с абелевым фактором $G / N$, то

$$
S(G, N)=\{\xi \in S(G) \mid N \leqslant \operatorname{Ker} \xi\}
$$

ТЕОРема 8. Пусть $C$ - свободный $\mathbb{Z} G$-цепной комплекс с конечным числом образующих в каждой размерности $i \leqslant n$ u $N$ - такая нормальная подгруппа группы $G$, что факторгруппа $G / N$ абелева. Тогда $C$ имеет конечный $n$-тип над $\mathbb{Z} N$ в том и только том случае, когда $S(G, N) \subset \Sigma^{n}(C)$.

Согласно работам [25], [26], теорема 7 следует из теоремы 8.

ДоказатеЛьство. Пусть $C$ имеет конечный $n$-тип над $\mathbb{Z} N$. Пусть $\xi \in$ $S(G, N)$, обозначим $Q=G / N$. Тогда $\xi$ индуцирует гомоморфизм, также обозначаемый через $\xi, \xi: Q \rightarrow \mathbb{R}$. Поскольку $\mathbb{Z} G$ является свободной над $\mathbb{Z} N$, то существует эквивалентность цепных комплексов $f: P \rightarrow C$ над $\mathbb{Z} N$, где $P_{j}$ конечно порожденные модули для всех $j \leqslant n$. Тогда из $f: \mathbb{Z} G_{\xi} \otimes_{\mathbb{Z} N} P \rightarrow \mathbb{Z} G_{\xi} \otimes_{\mathbb{Z} N} C$ видно, что $\mathbb{Z} G_{\xi} \otimes_{\mathbb{Z} N} C$ имеет конечный $n$-тип над $\mathbb{Z} G_{\xi}$. Кроме того, $\mathbb{Z} G_{\xi} \otimes_{\mathbb{Z} N} C \cong$ $\mathbb{Z} Q_{\xi} \otimes C$, где в цепном комплексе справа $\mathbb{Z} G_{\xi}$ действует диагонально. Изоморфизм задается отображением $g \otimes c \mapsto \pi(g) \otimes g c$, где $\pi: G \rightarrow Q$ является проекцией. Согласно [9; лемма 5.2], существует свободная резольвента $E_{*} \rightarrow \mathbb{Z}$ над $\mathbb{Z} Q_{\xi}$, которая конечно порождена в каждой размерности. Следовательно, каждый $E_{p} \otimes C$ имеет конечный $n$-тип над $\mathbb{Z} G_{\xi}$. Пусть $f: P_{p q} \rightarrow E_{p} \otimes C_{q}-$ соответствующее цепное отображение; заметим, что $P_{p q}$ - положительная степень $\mathbb{Z} G_{\xi} \otimes_{\mathbb{Z} N} P_{q}$, зависящая от ранга $E_{p}$. Поскольку $C-$ свободный над $\mathbb{Z} N$, то $\mathbb{Z} Q_{\xi} \otimes C \cong \mathbb{Z} G_{\xi} \otimes_{\mathbb{Z} N} C$ является свободным над $\mathbb{Z} G_{\xi}$, и можно предположить, что $f$ является цепной гомотопической эквивалентностью с обратным отображением $g_{q}: E_{p} \otimes C_{q} \rightarrow P_{p q}$. Обозначим через $L: E_{p} \otimes C_{q} \rightarrow E_{p} \otimes C_{q+1}$ цепную гомотопию $L: f g \simeq 1$.

Для $k \geqslant 0$ определим $F^{k}: P_{p q} \rightarrow E_{p-k} \otimes C_{q+k}$ формулой

$$
F^{k}=(L d)^{k} f
$$

где $d: E_{p} \rightarrow E_{p-1}$ - граница резольвенты. Определим $K^{i}: P_{p q} \rightarrow P_{p-i q-1+i}$ формулой

$$
K^{i}=g d F^{i-1}
$$

для $i \geqslant 1$. Положим $K^{0}=\partial: P_{p q} \rightarrow P_{p-1 q}$. 
Лемма 3. Пусть Ә обозначает границъ $\partial: P_{p q} \rightarrow P_{p q-1} u \partial: E_{p} \otimes C_{q} \rightarrow$ $E_{p} \otimes C_{q-1}$. Тогда $\partial F^{0}=F^{0} \partial$ и для любого $m \geqslant 1$ имеем

$$
\partial F^{m}+(-1)^{m+1} F^{m} \partial=\sum_{k=0}^{m-1}(-1)^{k} F^{k} K^{m-k}-d F^{m-1} .
$$

ДокАЗАТЕЛЬСТво непосредственно получается по индукции.

Лемма 4. Для $m \geqslant 0$ имеем

$$
\sum_{s=0}^{m}(-1)^{s} K^{m-s} K^{s}=0 .
$$

ДокАЗАТЕЛЬСтво. При $m=0$ это означает, что $\partial \partial=0$, поэтому допустим, что наше утверждение верно для $m \geqslant 0$. Тогда

$$
\begin{gathered}
\sum_{s=0}^{m+1}(-1)^{s} K^{m-s} K^{s}=g d F^{m} \partial+(-1)^{m+1} \partial g d F^{m}+\sum_{s=1}^{m}(-1)^{s} K^{m-s} K^{s} \\
=(-1)^{m+1}\left(g d \sum_{k=0}^{m-1}(-1)^{k} F^{k} K^{m-k}-g d d F^{m-1}\right) \\
+\sum_{s=1}^{m}(-1)^{s} g d F^{m-s-1} K^{s}=0
\end{gathered}
$$

согласно лемме 3 и поскольку $d d=0$. Лемма 4 доказана.

Определим цепной комплекс $T P$ формулой $T P_{k}=\bigoplus_{p+q=k} P_{p q}$ и $\delta: T P_{k} \rightarrow$ $T P_{k-1}$ формулой

$$
\delta=\sum_{s=0}^{\infty}(-1)^{p} K^{s}
$$

где $(-1)^{p}$ связано с $P_{p k-p}$. Из леммы 4 получаем $\delta \delta=0$. Определим также $T E_{k}=\bigoplus_{p+q=k} E_{p} \otimes C_{q}$ с дифференциалом $\delta=(-1)^{p}(\partial+d)$. Мы получаем цепной комплекс $F: T P \rightarrow T E$, полагая

$$
F=\sum_{k=0}^{\infty}(-1)^{k} F^{k}
$$

Тогда, как следует из лемм 3 и 4, $F$ является цепным отображением.

Используя фильтрации

$$
\left(T P^{(m)}\right)_{k}=\bigoplus_{p+q=k, p \leqslant m} P_{p q} \quad \text { и } \quad\left(T E^{(m)}\right)_{k}=\bigoplus_{p+q=k, p \leqslant m} E_{p} \otimes C_{q},
$$

видим, что $F$ индуцирует гомотопическую цепную эквивалентность

$$
\bar{F}: T P^{(m)} / T P^{(m-1)} \rightarrow T E^{(m)} / T E^{(m-1)},
$$

и из стандартных аргументов спектральных последовательностей следует, что $F$ является гомотопической цепной эквивалентностью. Аналогичное рас- 
суждение дает гомологическую эквивалентность $T E$ и $C$. Поскольку $T P_{k}-$ свободный конечно порожденный модуль над $\mathbb{Z} G_{\xi}$, получаем, что $C$ имеет конечный $n$-тип над $\mathbb{Z} G_{\xi}$.

Далее, предположим, что $S(G, N) \subset \Sigma^{n}(C)$.

Пусть $X_{i}$ является конечным $\mathbb{Z} G$-базисом в множестве $C_{i}, i \leqslant n$. Для $c \in C_{i}$ можно написать

$$
c=\sum_{j=1}^{m_{i}} n_{j}^{i} x_{j}^{i}, \quad n_{j}^{i} \in \mathbb{Z} G, \quad \partial c=\sum_{j=1}^{m_{i-1}} n_{j}^{i-1} x_{j}^{i-1}
$$

(здесь $x_{j}^{i}$ обозначают элементы множества $X_{i}$ ). Для $y \in \mathbb{Z} G$ определим supp $y$ как элементы $G$ с ненулевыми коэффициентами, а для $c \in C_{i}$ положим

$$
\operatorname{supp} c=\bigcup_{j=1}^{m_{i}} \operatorname{supp} n_{j}^{i} \cup \bigcup_{j=1}^{m_{i-1}} \operatorname{supp} n_{j}^{i-1} .
$$

В частности, $\operatorname{supp} \partial c \subset \operatorname{supp} c$. Носитель зависит от выбора базиса, но мы фиксируем базис раз и навсегда.

Обозначим $\pi: G \rightarrow G / N=Q$. Выбирая внутреннее произведение в $Q_{\mathbb{R}}=$ $\mathbb{R} \otimes Q$, мы получаем норму $\|\cdot\|$ в $Q_{\mathbb{R}}$ и можем рассматривать $S(G, N) \subset Q_{\mathbb{R}}$ как единичную сферу в этом нормированном векторном пространстве. Продолжим эту норму на $C$, положив

$$
\|c\|= \begin{cases}\max \{\|\pi(g)\| \mid g \in \operatorname{supp} c\}, & c \neq 0 \\ 0, & c=0 .\end{cases}
$$

Заметим, что мы полагаем $\|0\|=0$ - вопреки равенству $\|0\|=-\infty$ из [9]. Для $\xi \in S(G, N) \subset Q_{\mathbb{R}}$ мы также получаем $v_{\xi}$, полагая

$$
v_{\xi}(c)= \begin{cases}\min \{\langle\pi(g), \xi\rangle \mid g \in \operatorname{supp} c\}, & c \neq 0 \\ \infty, & c=0\end{cases}
$$

Для $a, b \in C$ положим

$$
\operatorname{diam}(a, b)=\max \{\|\pi(g)-\pi(h)\| \mid g \in \operatorname{supp} a, h \in \operatorname{supp} b\},
$$

считая, что $\operatorname{diam}(a, b)=0$, если $a$ или $b$ есть нуль. Наконец, для $r>0$ и $c \in C$ положим

$$
B_{r}(c)=\{d \in C \mid \operatorname{diam}(c, d) \leqslant r\} .
$$

Пусть $r>0$ задано. Тогда, поскольку $S(G, N) \subset \Sigma^{n}(C)$, для любого $\xi \in$ $S(G, N)$ мы можем найти цепное отображение $\varphi^{\xi}: C \rightarrow C$ и цепную гомотопию $H^{\xi}: 1 \simeq \varphi^{\xi}$ такие, что $v_{\xi}\left(\varphi^{\xi} c\right)-v_{\xi}(c) \geqslant 2 r$ для всех $c \in C$. Более того, существует такая открытая окрестность $U_{\xi}$ класса $\xi$ в $S(G, N)$, что

$$
v_{\eta}\left(\varphi^{\xi} c\right)-v_{\eta}(c) \geqslant r
$$

для всех $\eta \in U_{\xi}$ и $c \in C_{l}, l \leqslant n$. 
Поскольку $S(G, N)$ компактно, то для покрытия $S(G, N)$ достаточно конечного числа $U_{\xi}$; пусть $\left(U_{i}, \varphi^{i}, H^{i}\right), i=1, \ldots, k,-$ тройки, где $\left\{U_{i}\right\}$ покрывает $S(G, N), \varphi^{i}: C \rightarrow C$ - цепное отображение такое, что $v_{\eta}\left(\varphi^{i} c\right)-v_{\eta}(c) \geqslant r$ для всех $\eta \in U_{i}$ и $c \in C_{l}, l \leqslant n$, и $H^{i}: 1 \simeq \varphi^{i}$ - цепная гомотопия.

Поскольку мы имеем дело лишь с конечным числом цепных гомотопий, то существует такое $M \geqslant 0$, что $v_{\eta}(c)-v_{\eta}\left(H^{i} c\right) \leqslant M$ для всех $\eta \in U_{i}$ и $c \in C_{l}$, $l \leqslant n$. Более того, заменой цепной гомотопии $H^{i}$ на $H^{i}-\varphi^{i} H^{i}: 1 \simeq\left(\varphi^{i}\right)^{2}$ можно увеличить $r>0$ без увеличения $M>0$. Следовательно, можно предположить, что

$$
r>3 M n \text {. }
$$

Наконец, существует такое $L>0$, что

$$
\operatorname{diam}(x, x) \leqslant L, \quad \operatorname{diam}\left(x, \varphi^{i} x\right) \leqslant L, \quad \operatorname{diam}\left(x, H^{i} x\right) \leqslant L
$$

для всех $i=1, \ldots, k$ и всех $x \in X_{l}, l \leqslant n$, поскольку условий конечное число.

Заметим, что $C$ является свободным цепным комплексом над $\mathbb{Z} N$ и базис задается следующим образом: $T X_{i}=\left\{t x \mid t \in T, x \in X_{i}\right\}$, где $T \subset G$ - такое подмножество, что $\left.\pi\right|_{T}$ индуцирует биекцию из $T$ на $Q$.

Лемма 5. Если $S(G, N) \subset \Sigma^{n}(C)$, то существуют константы $r>0$, $M>0, A>0$, а такэе $\mathbb{Z} N$-цепное отображение $\psi: C \rightarrow C$ и $\mathbb{Z} N$-цепная гомотопия $K: 1 \simeq \psi$ такие, что при $m \leqslant n$ имеем $\psi_{m}(z)=z$, если $\|z\| \leqslant A$, a для $t x \in T X_{m} c\|t x\|>A$ выполнено неравенство

$$
\left\|\psi_{m}(t x)\right\| \leqslant\|t x\|-r .
$$

Кроме того, $\left\|K_{m}(z)\right\| \leqslant\|z\|+M$ для всех $z \in C_{m}$.

ДокАЗАТЕльСтво. Чтобы определить $\psi$ и $K$, мы определим их над $T X_{m}$. Пусть $r>0$ и $M>0$ такие же, как перед леммой 5 .

Лемма 6. Пусть $t x \in T X_{m} u\|t x\| \geqslant \max \left\{3 r / 4+L^{2} / r, L^{2} / M\right\}$. Пусть $i$ maкое, ито $\xi_{t} /\left\|\xi_{t}\right\| \in U_{i}$, где $\xi_{t}(g)=\langle\pi(g), \pi(t)\rangle$ для $g \in G$. Тогда

$$
\left\|\varphi^{i}(t x)\right\| \leqslant\|t x\|-\frac{1}{2} r, \quad\left\|H^{i}(t x)\right\| \leqslant\|t x\|+\frac{3}{2} M .
$$

ДокАЗАТЕЛЬСтво. Пусть для $g \in \operatorname{supp} t x$ выполнено равенство $\|\pi(g)\|=$ $\|t x\|$. Поскольку $\operatorname{diam}\left(t x, \varphi^{i}(t x)\right) \leqslant L$, то для $h \in \operatorname{supp} \varphi^{i}(t x)$

$$
\begin{aligned}
\|\pi(h)\|^{2} & \leqslant(\|\pi(g)\|-r)^{2}+L^{2}=\|\pi(g)\|^{2}-2 r\|\pi(g)\|+r^{2}+L^{2} \\
& \leqslant\|\pi(g)\|^{2}-r\|\pi(g)\|-\frac{3}{4} r^{2}-L^{2}+r^{2}+L^{2}=\left(\|\pi(g)\|-\frac{r}{2}\right)^{2}
\end{aligned}
$$

Следовательно, $\left\|\varphi^{i}(t x)\right\| \leqslant\|t x\|-r / 2$. Аналогично, используя неравенство $\|\pi(g)\| \geqslant L^{2} / M$, получаем $\left\|H^{i}(t x)\right\| \leqslant\|t x\|+3 M / 2$. Лемма 6 доказана.

Если $\|t x\|>L$, то $\pi(\operatorname{supp} t x) \subset Q_{\mathbb{R}}-\{0\}$. Обозначим через $p: Q_{\mathbb{R}}-\{0\} \rightarrow$ $S(G, N)$ стандартную ретракцию. Используя число Лебега накрытия $U_{1} \cup$ $\cdots \cup U_{k}$, можно найти такую константу $A^{\prime}>0$, что из $\|t x\|>A^{\prime}$ следует

$$
p\left(\pi\left(\operatorname{supp} B_{L^{\prime}}(t x)\right)\right) \subset U_{i}
$$

для некоторого $i$, где $L^{\prime}=\max \left\{3 r / 4+L^{2} / r, L^{2} / M\right\}+(2 n+1) L$. Пусть $A=A^{\prime}+L^{\prime}$. 
Определим $K_{0}: C_{0} \rightarrow C_{1}$ формулами $K_{0}(t x)=0$ при $\|t x\| \leqslant A$ и $K_{0}(t x)=$ $H_{0}^{i}(t x)$ при $\|t x\|>A$, где $i$ - наименьшее число, удовлетворяющее (4). Определим также $\psi_{0}: C_{0} \rightarrow C_{0}$ следующим образом:

$$
\psi_{0}(t x)= \begin{cases}t x, & \text { если }\|t x\| \leqslant A, \\ \varphi^{i}(t x), & \text { если }\|t x\|>A,\end{cases}
$$

где $i$ - опять наименьшее число, для которого имеет место (4). Отсюда следует, что $\partial K_{0}=1-\psi_{0}$. Из леммы 6 получаем

$$
\left\|\psi_{0}(t x)\right\| \leqslant\|t x\|-\frac{r}{2}, \quad\left\|K_{0}(t x)\right\| \leqslant\|t x\|+\frac{3}{2} M
$$

при $\|t x\|>A$. Кроме того,

$$
\operatorname{diam}\left(\psi_{0}(t x), t x\right) \leqslant L, \quad \operatorname{diam}\left(K_{0}(t x), t x\right) \leqslant L
$$

для всех базисных элементов $t x$.

Предположим по индукции, что для всех $j \leqslant m-1$ при $m \leqslant n$ мы имеем $K_{j}: C_{j} \rightarrow C_{j+1}$ и $\psi_{j}: C_{j} \rightarrow C_{j}$ такие, что

$$
\partial K_{j}+K_{j-1} \partial=1-\psi_{j}
$$

и

$$
\begin{aligned}
\left\|\psi_{j}(t x)\right\| & \leqslant\|t x\|-\frac{r}{2}+j \frac{3}{2} M, \\
\left\|K_{j}(t x)\right\| & \leqslant\|t x\|+\frac{3}{2} M(j+1)
\end{aligned}
$$

при $\|t x\|>A$ и

$$
\begin{aligned}
& \operatorname{diam}\left(\psi_{j}(t x), t x\right) \leqslant(2 j+1) L, \\
& \operatorname{diam}\left(K_{j}(t x), t x\right) \leqslant(2 j+1) L
\end{aligned}
$$

для всех базисных элементов $t x$.

Тогда определим $K_{m}: C_{m} \rightarrow C_{m+1}$ формулами $K_{m}(t x)=0$ при $\|t x\| \leqslant A$ и

$$
K_{m}(t x)=H_{m}^{i}(t x)-H_{m}^{i} K_{m-1} \partial(t x)
$$

при $\|t x\|>A$, где $i$ удовлетворяет (4). Непосредственная проверка показывает, что

$$
\left(\partial K_{m}+K_{m-1} \partial\right)(t x)=t x-\varphi_{m}^{i}(t x)+\varphi_{m}^{i}\left(K_{m-1} \partial t x\right)-H_{m-1}^{i} \psi_{m-1} \partial t x
$$

для $\|t x\|>A$. Следовательно, можно определить $\psi_{m}: C_{m} \rightarrow C_{m}$ как $\psi_{m}(t x)=$ $t x$ при $\|t x\| \leqslant A$ и

$$
\psi_{m}(t x)=\varphi_{m}^{i}(t x)-\varphi_{m}^{i}\left(K_{m-1} \partial t x\right)+H_{m-1}^{i} \psi_{m-1} \partial t x
$$

если $\|t x\|>A$. Тогда $\partial K_{m}+K_{m-1} \partial=1-\psi_{m}$. Поскольку

$$
\begin{aligned}
& \left\|\varphi_{m}^{i}(t x)-\varphi_{m}^{i}\left(K_{m-1} \partial t x\right)+H_{m-1}^{i} \psi_{m-1} \partial t x\right\| \\
& \quad \leqslant \max \left\{\left\|\varphi_{m}^{i}(t x)\right\|,\left\|\varphi_{m}^{i}\left(K_{m-1} \partial t x\right)\right\|,\left\|H_{m-1}^{i} \psi_{m-1} \partial t x\right\|\right\},
\end{aligned}
$$


легко видеть, что (5) имеет место при $j=m$. Здесь мы используем тот факт, что $\operatorname{diam}\left(K_{m-1} \partial t x, t x\right) \leqslant(2 m-1) L+L$ и, следовательно, лемма 6 применима ко всем базисным элементам, оказывающимся в $K_{m-1} \partial t x$ в силу выбора $A$.

Аналогично, оценки (6)-(8) верны при $j=m$. При $m>n$ положим $K_{m}=0$ и определим $\psi_{m}$ так, что

$$
\partial K_{m}+K_{m-1} \partial=1-\psi_{m}
$$

Поскольку тождественное отображение является цепным, мы получаем, что $\psi-$ цепное отображение. Заменяя $r$ на $r / 2-3 n M / 2$ и $M$ на $3 M(n+1) / 2$, мы получим лемму 5 . Заметим, что новое $r$ строго больше нуля согласно (3).

Лемма 7. Если $S(G, N) \subset \Sigma^{n}(C)$, то существуют свободный цепной комплекс $D$ над $\mathbb{Z} N$, у которого все $D_{i}$ конечно порождены при $i \leqslant n, u \mathbb{Z} N$-цепные отображения $a: D \rightarrow C, b: C \rightarrow D$ такие, что ab иепно-гомотопно тождественному отображению на $C$. Кроме того, можно считать, что $a: D_{i} \rightarrow C_{i}$ является вложением при $i \leqslant n$ u $D_{i}=C_{i}$ при $i>n$.

ДокАЗАтельство. Пусть $r, M, A>0, \psi$ и $K$ такие же, как в лемме 5 . Если $l$ положительное целое число, $B \geqslant 0$ и $z \in C_{m}$ при $m \leqslant n$, то

$$
\left\|\psi^{l}(z)\right\| \leqslant A+B, \quad \text { если }\|z\| \leqslant A+l r+B .
$$

Определим цепную гомотопию $\Phi: C \rightarrow C_{+1}$ над $\mathbb{Z} N$ следующим образом. При $s \leqslant n$ положим

$$
\Phi_{s}(t x)=\sum_{j=0}^{l} K_{s} \psi^{j} t x-K_{s} \psi^{j} \Phi_{s-1} \partial t x,
$$

где $l$ - такое целое число, что $\|t x\| \in(A+l r, A+(l+1) r]$, а при $s>n$ мы просто положим $\Phi_{s}=0$. Мы получаем $\mathbb{Z} N$-цепное отображение $\zeta: C \rightarrow C$, полагая $\zeta=1-\partial \Phi-\Phi \partial$. В частности, отображение $\zeta$ цепно-гомотопически эквивалентно тождественному.

Используя индукцию, мы видим, что

$$
\left\|\Phi_{s}(t x)\right\| \leqslant\|t x\|+(s+1) M \quad \text { при } s \leqslant n-1 .
$$

Отсюда следует неравенство

$$
\left\|\Phi_{s}(z)\right\| \leqslant\|z\|+(s+1) M
$$

для всех $z \in C_{s}$.

Мы утверждаем, что при $s \leqslant n$ имеет место неравенство

$$
\|\zeta(z)\| \leqslant A+s M
$$

для всех $z \in C_{s}$.

При $s=0$ это верно согласно (9), поскольку $\zeta(t x)=\psi^{l+1}(t x)$, если $\|t x\| \in$ $(A+l r, A+(l+1) r]$. 
Заметим, что

$$
\begin{aligned}
\left(\partial \Phi_{s}+\right. & \left.\Phi_{s-1} \partial\right)(t x)=\sum_{j=0}^{l}\left(K_{s} \psi^{j} t x-K_{s} \psi^{j} \Phi_{s-1} \partial t x\right)+\Phi_{s-1} \partial(t x) \\
= & \sum_{j=0}^{l}\left(\partial K_{s} \psi^{j}+K_{s-1} \partial \psi^{j}\right)(t x)-\sum_{j=0}^{l}\left(\partial K_{s} \psi^{j} \Phi_{s-1}+K_{s-1} \partial \psi^{j} \Phi_{s-1}\right)(\partial t x) \\
& \quad+\sum_{j=0}^{l}\left(K_{s-1} \psi^{j} \partial \Phi_{s-1} \partial t x-K_{s-1} \partial \psi^{j} t x\right)+\Phi_{s-1} \partial t x \\
= & t x-\psi^{l+1} t x-\Phi_{s-1} \partial t x+\psi^{l+1} \Phi_{s-1} \partial t x \\
& \quad-\sum_{j=0}^{l} K_{s-1} \partial \psi^{j} t x+\Phi_{s-1} \partial t x+\sum_{j=0}^{l}\left(K_{s-1} \psi^{j} \partial t x-K_{s-1} \psi^{j} \zeta \partial t x\right) \\
= & t x-\psi^{l+1} t x+\psi^{l+1} \Phi_{s-1} \partial t x-\sum_{j=0}^{l} K_{s-1} \psi^{j} \zeta \partial t x .
\end{aligned}
$$

Отсюда следует, что

$$
\zeta_{s}(t x)=\psi^{l+1} t x-\psi^{l+1} \Phi_{s-1} \partial t x+\sum_{j=0}^{l} K_{s-1} \psi^{j} \zeta_{s-1} \partial t x .
$$

Используя индукцию, мы получаем, снова ввиду (9), что (10) имеет место.

Определим цепной комплекс $D$ следующим образом:

$$
D_{s}=\left\{z \in C_{s} \mid\|z\| \leqslant A+n M\right\}
$$

при $s \leqslant n$; при $s>n$ положим $D_{s}=C_{s}$. Граничное отображение $\partial_{D}: D_{n+1} \rightarrow D_{n}$ задается формулой $\partial_{D}=\zeta$ ○ $\partial_{C}$. Мы можем определить цепное отображение $b: C \rightarrow D$ как $\zeta$ при $s \leqslant n$ и тождественное отображение при $s>n$, а цепное отображение $a: D \rightarrow C$ как вложение при $s \leqslant n$ и $\zeta$ при $s>n$. Тогда отображение $a b=\zeta: C \rightarrow C$ цепно-гомотопно тождественному. Поскольку только конечное число $q \in Q$ удовлетворяют неравенству $\|q\| \leqslant A+n M$, мы получаем, что $D_{s}$ является конечно порожденным свободным модулем над $\mathbb{Z} N$ (ср. [9; п. 5.4]). Это завершает доказательство леммы 7.

Для завершения доказательства теоремы 8 заметим, что согласно лемме 7 цепной комплекс $C$ доминируется над $\mathbb{Z} N$ цепным комплексом $D$ с конечно порожденными свободными модулями $D_{i}$ при $i \leqslant n$. По стандартной конструкции (cp. [27; §3] или [26]), существует $\mathbb{Z} N$-цепной комплекс $E$, цепно-гомотопически эквивалентный $C$, такой, что $E_{i}$ являются конечно порожденными модулями при $i \leqslant n$.

СЛЕДСТВИЕ 4. Пусть $C$ - конечно порожденный свободный $\mathbb{Z} G$-цепной комплекс с $C_{i}=0$ при $i>n$. Тогда $C$ чепно-гомотопически эквивалентен конечно порожденному проективному $\mathbb{Z} N$-цепному комплексу $D$, в котором $D_{i}=0$ при $i>n$ тогда и толъко тогда, когда $S(G, N) \subset \Sigma^{n}(C)$. 
ДокАЗАТЕЛьство. Если $C$ цепно-гомотопически эквивалентен над $\mathbb{Z} N$ конечно порожденному $\mathbb{Z} N$-комплексу, то он имеет, по определению, конечный $n$-тип над $\mathbb{Z} N$, следовательно, $S(G, N) \subset \Sigma^{n}(C)$ по теореме 8.

Если $S(G, N) \subset \Sigma^{n}(C)$, то по лемме 7 существует цепной комплекс $D^{\prime}$, конечно порожденный и свободный над $\mathbb{Z} N$ с $D_{i}^{\prime}=0$ при $i>n$, который доминирует $C$. Согласно [27; предложение 3.1$]$, требуемый цепной комплекс $D$ существует.

\section{8. Подвижность гомологических классов}

Напомним, что, согласно предложению 5 , свойство зануления гомологий Новикова-Сикорава в случае универсального накрытия определяется инвариантом $\Sigma^{k}(X, \mathbb{Z})$. Сейчас мы хотим более подробно рассмотреть случаи других накрытий, в частности накрытий абелевой группой монодромии.

В этом разделе $R$ - кольцо, хотя в основном мы будем иметь в виду случай $R=\mathbb{Z}$ или $R=\mathbf{k}$, где $\mathbf{k}-$ поле.

Пусть $p: \bar{X} \rightarrow X$ - регулярное накрытие, где $X$ - опять конечный связный клеточный комплекс. Введем обозначение $G=\pi_{1}(X) / \pi_{1}(\bar{X})$. Напомним, что $S(X, \bar{X})$ состоит из таких $\xi \in S(X)$, что $p^{*} \xi=0$. Для такого класса $\xi$ можно определить функцию высоты $h: \bar{X} \rightarrow \mathbb{R}$ и окрестности $N \subset \bar{X}$ бесконечности относительно $\xi$, как в случае универсального накрытия.

ОПРЕДЕЛЕНИЕ 14. Будем говорить, что класс гомологий $z \in H_{q}(\bar{X} ; R)$ является удаляемым на бесконечность в $\bar{X}$ относительно $\xi \in S(X, \bar{X})$, если $z$ можно реализовать сингулярным циклом в любой окрестности $N$ бесконечности относительно $\xi$.

Другими словами, $z \in H_{q}(\bar{X} ; R)$ является удаляемым на бесконечность относительно $\xi$, если $z$ является элементом

$$
\bigcap_{N} \operatorname{Im}\left(H_{q}(N ; R) \rightarrow H_{q}(\bar{X} ; R)\right),
$$

где пересечение берется по всем окрестностям бесконечности относительно $\xi$.

Заметим, что мы имеем обратную систему $R$-модулей

$$
\left\{H_{q}(\bar{X}, N ; R) \leftarrow H_{q}\left(\bar{X}, N^{\prime} ; R\right)\right\},
$$

которая пробегает все окрестности $N^{\prime} \subset N$ бесконечности относительно $\xi$. Следовательно, $z$ является удаляемым на бесконечность относительно $\xi$ тогда и только тогда, когда

$$
z \in \operatorname{Ker}\left(H_{q}(\bar{X} ; R) \rightarrow \lim _{\longleftarrow} H_{q}(\bar{X}, N ; R)\right) .
$$

Так же, как и для целочисленных коэффициентов, мы можем определить кольцо Новикова-Сикорава $\widehat{R G}_{\xi}$ с коэффициентами в произвольном кольце $R$. Поскольку это кольцо представляется как обратный предел, стандартные методы дают следующую точную последовательность:

$$
0 \rightarrow \lim ^{1} H_{q+1}(\bar{X}, N ; R) \rightarrow H_{q}\left(X ; \widehat{R G}_{-\xi}\right) \rightarrow \underset{\lim }{\longleftarrow} H_{q}(\bar{X}, N ; R) \rightarrow 0
$$

(см. [22] или [16] для более детального ознакомления). 
Дадим простой критерий для проверки удаляемости класса $z \in H_{i}(\bar{X} ; R)$ на бесконечность.

ОПРЕДЕЛЕНИЕ 15. Будем говорить, что элемент $\Delta \in R G$ имеет $\xi$-минимальнъй коэфбициент 1 , если $\Delta=1-y$, где $y=\sum a_{j} g_{j} \in R G$ и каждый $g_{j} \in G$ удовлетворяет неравенству $\xi\left(g_{j}\right)>0$.

Такое $\Delta$ обратимо над $\widehat{R G}_{-\xi}$. Следовательно, если $\Delta \cdot z=0 \in H_{q}(\bar{X} ; R)$, мы получаем, что образ $z$ в $H_{q}\left(X ; \widehat{R G}_{-\xi}\right)$ нулевой, и, согласно $(11), z$ является удаляемым на бесконечность. При некоторых условиях свойство “ $\Delta$ имеет $\xi$-минимальный коэффициент 1" является необходимым для удаляемости на бесконечность. Следующая теорема взята из [22], [28].

ТЕОрема 9. Пустъ $X$ - конечный связный клеточный комплекс, и пусть $p: \bar{X} \rightarrow X-$ регулярное накрытие с группой монодромии $G \cong \mathbb{Z}^{r}$. Предположим, что $\xi \in S(X, \bar{X})$ индуцирует ингективный гомоморфизм $G \rightarrow \mathbb{R} u R-$ кольцо $\mathbb{Z}$ или поле. При $z \in H_{q}(\bar{X} ; R)$ следующие условия равносильны:

1) z удаляем на бесконечность относительно $\xi$;

2) $i_{*}(z)=0 \in H_{q}\left(X ; \widehat{R G}_{-\xi}\right)$, где $i_{*}: H_{q}(\bar{X} ; R) \cong H_{q}(X ; R G) \rightarrow H_{q}\left(X ; \widehat{R G}_{-\xi}\right)$ является заменой коэфбициентов;

3) существует элемент $\Delta \in R G$ с -минимальным коэббициентом 1 такой, что $\Delta \cdot z=0$.

Когда кольцо $R$ является полем, условие 3 ) эквивалентно существованию ненулевого $\Delta \in R G$ с $\Delta \cdot z=0 \in H_{q}(\bar{X} ; R)$, поскольку мы можем найти такие $r \in R$ и $g \in G$, что $\Delta \cdot r g$ имеет $\xi$-минимальный коэффициент 1 .

Заметим, что свойство " $\Delta \in R G$ имеет $\xi$-минимальный коэффициент 1 " является открытым свойством в $\xi \in S(X, \bar{X})$; в частности, если $z$ удаляем на бесконечность относительно $\xi$, то при условиях теоремы 9 он также удаляем на бесконечность относительно близких $\xi^{\prime}$.

Эквивалентность условий 1) и 2) будет доказана, если показать, что член $\lim ^{1}$ в (11) нулевой. Это сделано в [22], [28] в условиях теоремы 9. Используя [29; теорема 1.3], можно показать, что член $\lim ^{1}$ в (11) на самом деле обращается в нуль для любого абелева накрытия и любого нётерова кольца $R$.

Теорема 9 является важным элементом для получения нижней оценки $\operatorname{cat}(X, \xi)$ и $\operatorname{cat}^{1}(X, \xi)$ через произведения. Подробности см. в [30], [28].

\section{9. Функциональное пространство путей в бесконечность}

Если $\gamma:[0, \infty) \rightarrow X-$ отображение, то мы можем поднять его на универсальное накрытие, $\widetilde{\gamma}:[0, \infty) \rightarrow \widetilde{X}$. Мы хотим найти отображения, которые при поднятии дают пути в бесконечность относительно заданного класса $\xi$ (ср. определение 7). Это не зависит от конкретного поднятия отображения $\gamma$.

Введем множество

$$
\mathscr{C}_{\xi}(X)=\{\gamma:[0, \infty) \rightarrow X \mid \widetilde{\gamma}-\text { путь в бесконечность относительно } \xi\} .
$$

Оно снабжается следующей топологией: для $a, b \in[0, \infty)$ и открытого множества $U$ пространства $X$ положим

$$
W(a, b ; U)=\left\{\gamma \in \mathscr{C}_{\xi}(X) \mid \gamma([a, b]) \subset U\right\}
$$


а при $a, A \in[0, \infty)$ пусть

$$
W(a, A)=\left\{\gamma \in \mathscr{C}_{\xi}(X) \mid h_{\xi} \widetilde{\gamma}(t)-h_{\xi} \widetilde{\gamma}(0)>A \text { для любого } t \geqslant a\right\} .
$$

Эти множества образуют базу топологии на $\mathscr{C}_{\xi}(X)$. Заметим, что множества $W(a, b ; U)$ задают компактно-открытую топологию на $\mathscr{C}_{\xi}(X)$, тогда как множества $W(a, A)$ задают "поведение на бесконечности".

Функция $e: \mathscr{C}_{\xi}(X) \rightarrow X$, заданная формулой $e(\gamma)=\gamma(0)$, является расслоением, и для $x_{0} \in X$ получаем слой

$$
\mathscr{M}_{\xi}=\left\{\gamma \in \mathscr{C}_{\xi}(X) \mid \gamma(0)=x_{0}\right\}
$$

(cp. [11]).

ЗАмЕЧАНИЕ 4. Относительно компактно-открытой топологии слой $\mathscr{M}_{\xi}$ является стягиваемым. Чтобы доказать это, выберем $\gamma_{\infty} \in \mathscr{M}_{\xi}$. Для любого $\gamma \in \mathscr{M}_{\xi}$ и $t \in[0, \infty)$ зададим $\gamma_{t} \in \mathscr{M}_{\xi}$ как

$$
\gamma_{t}(s)= \begin{cases}\gamma_{\infty}(s), & 0 \leqslant s \leqslant t \\ \gamma_{\infty}(2 t-s), & t \leqslant s \leqslant 2 t \\ \gamma(s-2 t), & 2 t \leqslant s\end{cases}
$$

Легко видеть, что отображение $H: \mathscr{M}_{\xi} \times[0, \infty] \rightarrow \mathscr{M}_{\xi}$, заданное формулой $H(\gamma, t)=\gamma_{t}$, является непрерывным в компактно-открытой топологии и, следовательно, задает стягивание. Однако $H$ уже не является непрерывным, если множества $W(a, A)$ открыты в $\mathscr{M}_{\xi}$.

ЗАмЕчАНИЕ 5. Дадим иную интерпретацию топологии на пространстве $\mathscr{M}_{\xi}$. Пусть $\widetilde{X}_{\infty}=\tilde{X} \cup\{\infty\}$, т. е. мы добавили к $\widetilde{X}$ точку $\infty$; топология на $\widetilde{X}_{\infty}$ задается открытыми множествами пространства $\widetilde{X}$ и множествами $N \cup\{\infty\}$, где $N$ открытая окрестность бесконечности относительно $\xi$ (в смысле раздела 2).

Введем множество

$$
\mathscr{P}\left(\widetilde{X}_{\infty}\right)=\left\{\gamma:[0, \infty] \rightarrow \widetilde{X}_{\infty} \mid \gamma(\infty)=\infty\right\}
$$

и снабдим его компактно-открытой топологией. Это обычное пространство путей, где бесконечность $\infty$ является отмеченной точкой. Пространство $\mathscr{C}_{\xi}$ путей в бесконечность относительно класса $\xi$ можно отождествить с подпространством в пространстве $\mathscr{P}\left(\widetilde{X}_{\infty}\right)$, состоящим из таких путей $\gamma$, что $\gamma([0, \infty)) \subset \widetilde{X}$. Аналогичным образом, $\mathscr{M}_{\xi}$ можно отождествить с подпространством пространства $\Omega\left(\widetilde{X}_{\infty}\right)=e^{-1}\left(\left\{\tilde{x}_{0}\right\}\right)$, где $\tilde{x}_{0} \in p^{-1}\left(\left\{x_{0}\right\}\right) \subset \widetilde{X}$ и $e: \mathscr{P}\left(\widetilde{X}_{\infty}\right) \rightarrow \widetilde{X}_{\infty}$ - обычное расслоение. Заметим, что $\mathscr{C}_{\xi}$ является накрывающим для $\mathscr{C}_{\xi}(X)$ с группой монодромии $\pi_{1}(X)$.

Имея $\gamma_{0} \in \mathscr{M}_{\xi}$, мы хотим изучить гомотопические группы $\pi_{k}\left(\mathscr{M}_{\xi}, \gamma_{0}\right)$ при $k \geqslant 0$. Для этого введем отображение $g:\left(S^{k}, *\right) \rightarrow\left(\mathscr{M}_{\xi}, \gamma_{0}\right)$. Оно поднимается до отображения $\phi_{g}: S^{k} \times[0, \infty) \rightarrow X$ по формуле $\phi_{g}(x, t)=g(x)(t)$, и, поскольку $\phi_{g}(x, 0)=x_{0}$, до отображения $\Phi_{g}: \mathbb{R}^{k+1} \rightarrow X$ такого, что $\Phi_{g}(x \cdot t)=\phi(x, t)$ для $x \in S^{k} \subset \mathbb{R}^{k+1}$. Если предположить, что $* \in S^{k}$ соответствует $(1,0, \ldots, 0) \in$ $\mathbb{R}^{k+1}$, то $\Phi_{g}(t, 0, \ldots, 0)=\gamma_{0}(t)$. Более того, если поднять $\Phi_{g}$ до отображения 
$\widetilde{\Phi}_{g}: \mathbb{R}^{k+1} \rightarrow \widetilde{X}$, получим, что $h_{\xi} \circ \widetilde{\Phi}(x) \rightarrow \infty$ при $|x| \rightarrow \infty$. Гомотопия между двумя отображениями $g_{0}, g_{1}:\left(S^{k}, *\right) \rightarrow\left(\mathscr{M}_{\xi}, \gamma_{0}\right)$ относительно отмеченной точки соответствует гомотопии $\Phi: \mathbb{R}^{k+1} \times[0,1] \rightarrow X$ между $\Phi_{g_{0}}$ и $\Phi_{g_{1}}$ относительно $[0, \infty) \times\{0\}$ такой, что $h_{\xi} \circ \widetilde{\Phi}(x, s) \rightarrow \infty$ при $|x| \rightarrow \infty$ равномерно по $s \in[0,1]$ для поднятия $\widetilde{\phi}$.

Предположим, что $\left(N_{i}\right)_{i \geqslant 0}$ - система окрестностей бесконечности относительно $\xi$ такая, что $N_{i} \subset N_{i-1}$ для всех $i$ и $\bigcap_{i \geqslant 0} N_{i}=\varnothing$. Пусть $\tilde{x}_{0} \in \tilde{X}-$ поднятие $x_{0} \in X$. Пусть также $\widetilde{\gamma}_{0}:[0, \infty) \rightarrow \widetilde{X}$ - такое поднятие $\gamma_{0}$, что $\widetilde{\gamma}_{0}(0)=\tilde{x}_{0}$. Возьмем последовательность $t_{i}>0$ такую, что $t_{i+1}>t_{i}$ для всех $i \geqslant 0$ и $\widetilde{\gamma}_{0}(t) \in N_{i}$ для любого $t \geqslant t_{i}$. Такая последовательность существует по определению $\gamma_{0} \in \mathscr{M}_{\xi}$. Пусть

$$
y_{i}=\widetilde{\gamma}_{0}\left(t_{i}\right) \in N_{i}
$$

- последовательность отмеченных точек окрестностей $N_{i}$ для всех $i \geqslant 0$. Имеем естественные гомоморфизмы $\chi_{i}: \pi_{k}\left(\widetilde{X}, N_{i+1}, y_{i+1}\right) \rightarrow \pi_{k}\left(\widetilde{X}, N_{i}, y_{i}\right)$, индуцированные вложениями, где изменение отмеченной точки задается путем $\left.\widetilde{\gamma}_{0}\right|_{\left[t_{i}, t_{i+1}\right]}$. Они поднимаются на обратную систему $\left(\chi_{i}: \pi_{k}\left(\widetilde{X}, N_{i+1}, y_{i+1}\right) \rightarrow \pi_{k}\left(\widetilde{X}, N_{i}, y_{i}\right)\right)$ для всех $k \geqslant 0$. Заметим, что она является обратной системой с отмеченными точками для всех $k \leqslant 1$.

Для любых $i \geqslant 0$ и $k \geqslant 0$ определим $\varphi_{i}: \pi_{k}\left(\mathscr{M}_{\xi}, \gamma_{0}\right) \rightarrow \pi_{k+1}\left(\widetilde{X}, N_{i}, y_{i}\right)$ следующим образом. Для данного $g:\left(S^{k}, *\right) \rightarrow\left(\mathscr{M}_{\xi}, \gamma_{0}\right)$ определим $\varphi_{g}:\left(D^{k+1}, S^{k}, *\right) \rightarrow$ $\left(\widetilde{X}, N_{i}, y_{i}\right)$ формулой $\varphi_{g}(x \cdot t)=\widetilde{\Phi}_{g}(\lambda(x) \cdot x \cdot t)$ для $x \in S^{k}$ и $t \in[0,1]$, где $\lambda: S^{k} \rightarrow(0, \infty)$ - такое отображение, что $\lambda(*)=t_{i}$, и для любого $x \in S^{k}$ имеем $g(x)(t) \in N_{i}$ при всех $t \geqslant \lambda(x)$. Очевидно, что гомотопический класс $\varphi_{g}$ не зависит от выбора конкретного $\lambda$. Мы используем $\widetilde{\Phi}_{g}$ и ограничим его на достаточно большой шар в $\mathbb{R}^{k+1}$.

Это индуцирует отображение $\varphi_{i}: \pi_{k}\left(\mathscr{M}_{\xi}, \gamma_{0}\right) \rightarrow \pi_{k+1}\left(\widetilde{X}, N_{i}, y_{i}\right)$, при этом очевидно, что $\chi_{i} \varphi_{i+1}=\varphi_{i}$. Следовательно, мы получаем групповой гомоморфизм

$$
\varphi: \pi_{k}\left(\mathscr{M}_{\xi}, \gamma_{0}\right) \rightarrow \lim _{\longleftarrow} \pi_{k+1}\left(\widetilde{X}, N_{i}, y_{i}\right)
$$

для $k \geqslant 1$ и отображение пространств с отмеченными точками для $k=0$.

Далее, определим отображение $\psi^{\prime}: \prod_{i \geqslant 0} \pi_{k+2}\left(\widetilde{X}, N_{i}, y_{i}\right) \rightarrow \pi_{k}\left(\mathscr{M}_{\xi}, \gamma_{0}\right)$ и положим $a_{i} \in \pi_{k+2}\left(\widetilde{X}, N_{i}, y_{i}\right)$. Представим $a_{i}$ отображением $g_{i}:\left(D^{k+2}, S^{k+1}, *\right) \rightarrow$ $\left(\widetilde{X}, N_{i}, y_{i}\right)$. Далее, пусть $G^{\prime}: \mathbb{R}^{k+1} \rightarrow \widetilde{X}$ задано как $G^{\prime}(x \cdot t)=\widetilde{\gamma}_{0}(t)$ для $x \in S^{k}$ и $t \in[0, \infty)$. Выберем диски $B\left(t_{i}\right)$ с центрами в точках $\left(-t_{i}, 0\right) \in$ $\mathbb{R}^{k+1}$ настолько малыми, чтобы они попарно не пересекались. Мы используем $\left(-t_{i}, 0\right)$ как центр, поскольку мы хотим изменить $G^{\prime}$ на $B\left(t_{i}\right)$, не изменяя его на $[0, \infty) \times\{0\} \subset \mathbb{R}^{k+1}$. Следовательно, мы гомотопируем $G^{\prime}$ в отображение $G$ относительно $\mathbb{R}^{k+1}-\bigcup B^{\prime}\left(t_{i}\right)$ так, что $G$ является постоянным $\widetilde{\gamma}_{0}\left(t_{i}\right)$ на $B\left(t_{i}\right)$ для всех $i \geqslant 0$. Здесь $B^{\prime}\left(t_{i}\right)$ - чуть бо́льшие диски, по-прежнему попарно не пересекающиеся.

Если ограничить отображение $g_{i}$ на $S^{k+1}$, его можно рассматривать как отображение $\tilde{g}_{i}:\left(D^{k+1}, S^{k}\right) \rightarrow\left(N_{i}, y_{i}\right)$. Теперь мы можем заменить $G$ на такое отображение $\widetilde{G}: \mathbb{R}^{k+1} \rightarrow \widetilde{X}$, что $\left.\widetilde{G}\right|_{B\left(t_{i}\right)} \equiv \tilde{g}_{i}$ и $\widetilde{G}$ совпадает с $G$ в остальной 
части. Очевидно, что $\widetilde{G}$ индуцирует отображение $g:\left(S^{k}, *\right) \rightarrow\left(\mathscr{M}_{\xi}, \gamma_{0}\right)$ и это определяет отображение

$$
\psi^{\prime}: \prod_{i \geqslant 0} \pi_{k+2}\left(\tilde{X}, N_{i}, y_{i}\right) \rightarrow \pi_{k}\left(\mathscr{M}_{\xi}, \gamma_{0}\right) .
$$

Заметим, что если $g_{i}^{\prime}:\left(D^{k+2}, S^{k+1}, *\right) \rightarrow\left(\widetilde{X}, N_{i}, y_{i}\right)$ также представляют $a_{i}$ для любого $i \geqslant 0$, то $\left.g_{i}\right|_{S^{k+1}}$ гомотопно $\left.g_{i}^{\prime}\right|_{S^{k+1}}$ внутри $N_{i}$, следовательно, результирующие отображения $g$ и $g^{\prime}$ задают один и тот же элемент в $\pi_{k}\left(\mathscr{M}_{\xi}, \gamma_{0}\right)$. Стоит отметить, что $\widetilde{G}$ гомотопно $G$ относительно $[0, \infty) \times\{0\}$, но результирующая функция $H: S^{k} \times[0,1] \rightarrow \mathscr{M}_{\xi}$ не обязана быть непрерывной, поскольку $g_{i}$ являются нуль-гомотопными в $\widetilde{X}$, но не обязательно в смысле определения 4 .

Напомним определение предела для нашей обратной системы. Две последовательности $\left(a_{i}\right),\left(b_{i}\right) \in \prod_{i \geqslant 0} \pi_{k+2}\left(\tilde{X}, N_{i}, y_{i}\right)$ называются эквивалентными, если существует такая последовательность $\left(c_{i}\right) \in \prod_{i \geqslant 0} \pi_{k+2}\left(\widetilde{X}, N_{i}, y_{i}\right)$, что $b_{i}=$ $c_{i} \cdot a_{i} \cdot \chi_{i}\left(c_{i+1}\right)$ для всех $i \geqslant 0$. Тогда $\lim ^{1} \pi_{k+2}\left(\widetilde{X}, N_{i}, y_{i}\right)$ является множеством классов эквивалентности. Для $k \geqslant 1$ оно имеет структуру абелевой группы, но при $k=0$ имеем только множество с отмеченной точкой.

Легко видеть, что $\psi^{\prime}$ индуцирует отображение

$$
\psi: \lim ^{1} \pi_{k+2}\left(\tilde{X}, N_{i}, y_{i}\right) \rightarrow \pi_{k}\left(\mathscr{M}_{\xi}, \gamma_{0}\right),
$$

которое является гомоморфизмом при $k \geqslant 1$ и отображением множеств с отмеченной точкой при $k=0$.

ПРЕДЛОЖЕНИЕ 9. При вышеприведенных обозначениях существует короткая точная последовательность

$$
1 \rightarrow \lim ^{1} \pi_{k+2}\left(\tilde{X}, N_{i}, y_{i}\right) \stackrel{\psi}{\longrightarrow} \pi_{k}\left(\mathscr{M}_{\xi}, \gamma_{0}\right) \stackrel{\varphi}{\longrightarrow} \lim _{\longleftarrow} \pi_{k+1}\left(\tilde{X}, N_{i}, y_{i}\right) \rightarrow 1,
$$

которая является короткой точной последовательностью абелевых групп при $k \geqslant 1$ и множеств с отмеченной точкой при $k=0$. Если $k=0$, то $\psi$ ингективна.

Доказательство стандартное (см. [16]), и мы его опускаем. Отметим схожесть между последовательностью предложения 9 и последовательностью из (11).

Используя это, можно дать другое, эквивалентное определение $\xi \in \Sigma^{k}(X)$.

ПреДЛОЖЕНИЕ 10. Пусть $X$ - конечный связный клеточный комплекс, $\xi \in H^{1}(X ; \mathbb{R})$ - ненулевой класс и $k \geqslant 1$. Тогда $\xi \in \Sigma^{k}(X)$ в том и только том случае, когда $\mathscr{M}_{\xi}$ является $(k-1)$-связным.

ДокАЗАТЕЛЬСтво. Если $\mathscr{M}_{\xi}$ является $(k-1)$-связным, то по предложению 9 имеем обратную систему $\left\{\pi_{l}(\tilde{X}, N)\right\}$, которая про-тривиальна при $l \leqslant k$, что согласно предложению 2 дает $\xi \in \Sigma^{k}(X)$.

Чтобы доказать утверждение в части “только в том случае", мы должны побеспокоиться о $\lim ^{1} \pi_{k+1}\left(\widetilde{X}, N_{i}\right)$. Но согласно следующей лемме система $\left\{\pi_{k+1}(\widetilde{X}, N)\right\}$ является полустабильной, следовательно, $\lim ^{1}$-член обращается в нуль. Из предложения 9 следует, что $\mathscr{M}_{\xi}$ является $(k-\overleftarrow{1})$-связным. 
ЛЕмма 8. Пусть $X$ - конечный связный клеточный комплекс, класс $\xi \in$ $H^{1}(X ; \mathbb{R})$ - ненулевой и $h_{\xi}: \widetilde{X} \rightarrow \mathbb{R}-$ функция высоты. Если $\xi \in \Sigma^{k}(X)$, то система $\left\{\pi_{k+1}(\widetilde{X}, N)\right\}$ является полустабильной.

ДоКАЗАТЕЛЬСТво. Используем гомотопию из предложения 2,4$)$, чтобы удалить любую $k$-сферу в $N$ достаточно далеко; это дает полустабильность, поскольку процедура может быть проделана в окрестности $N^{\prime}$, несколько большей, чем $N$ (зависит только от $H$ ).

\section{Приложение А. Сигма-инварианты цепных комплексов}

В этом приложении мы покажем, как $\Sigma^{k}(C)$ связан с критерием, в котором участвуют цепные гомотопии на $C$.

Если цепной комплекс $C$ состоит из плоских $R$-модулей, мы имеем следующий критерий.

ПРЕДЛОЖЕНИЕ 11. Пусть $C$ - цепной комплекс плоских $R$-модулей u $n-$ неотрицательное целое число. Тогда следующие условия эквивалентнь:

1) С имеет конечный $n$-тип;

2) для любого множества индексов $J$ естественное отображение

$$
H_{k}\left(C, \prod_{J} R\right) \rightarrow \prod_{J} H_{k}(C)
$$

является изоморфизмом при $k<n$ и эпиморфизмом при $k=n$.

По существу это теорема 2 из [31], но мы разрешаем комплексу $C$ быть плоским и не обязательно проективным, поэтому мы получаем только гомологический критерий. Доказательство проходит для плоских модулей.

Под фильтрацией в $C$ мы понимаем семейство $\left\{C^{\alpha}\right\}_{\alpha \in \mathscr{A}}$ цепных подкомплексов, где $\mathscr{A}$ - направленное множество, $C^{\alpha} \subset C^{\beta}$ для $\alpha \leqslant \beta$ и $C=\bigcup C^{\alpha}$.

Имея фильтрацию, определяем $D^{\alpha}=C / C^{\alpha}$.

Справедлив следующий аналог теоремы 2.2. работы [32].

Теорема 10. Пусть $C$ - цепной комплекс над $R$ с фильтрацией $\left\{C^{\alpha}\right\}_{\alpha \in \mathscr{A}}$ конечного $n$-типа комплексами $C^{\alpha}$ плоских $R$-модулей. Тогда $C$ имеет конечный $n$-тип в том и только том случае, когда прямая система $\left\{H_{i}\left(D^{\alpha}\right)\right\}$ существенно тривиальна при $i \leqslant n$.

ДокАЗАТЕЛЬство аналогично доказательству теоремы 2.2 в [32], но надо использовать предложение 11. Детали мы опустим.

Пусть $G$ - конечно порожденная группа и $\xi: G \rightarrow \mathbb{R}$ - ненулевой гомоморфизм. Пусть $C$ - свободный конечно порожденный в каждой размерности цепной комплекс над $\mathbb{Z} G$. Для заданного нормирования на $C$, продолжающего $\xi$, определим подкомплекс $C^{\xi}=\{x \in C \mid v(x) \geqslant 0\}$. Нормирования определяются своими значениями на базисных элементах, поэтому легко видеть, что $C^{\xi}$ является конечно порожденным свободным цепным комплексом над $\mathbb{Z} G_{\xi}$ (cp. [9; лемма 3.1]). Для $g \in G$ мы также можем рассмотреть подкомплекс $g C^{\xi} \subset C$, который изоморфен $C^{\xi}$. Обозначим $D^{g}=C / g C^{\xi}$. 
Используя теорему 10, получаем следующий результат.

ПрЕДЛОЖЕНИЕ 12. Пустъ $C$ - свободный конечно порожденный в каждой размерности цепной комплекс над $\mathbb{Z} G, v$ - нормирование, продолжающее $\xi$, и - неотрицательное целое число. Тогда следующие свойства эквивалентны:

1) $\xi \in \Sigma^{n}(C)$;

2) прямая система $\left\{H_{i}\left(D^{g}\right)\right\}$ существенно тривиальна при $i \leqslant n$;

3) существует цепное отображение $\varphi: C \rightarrow C$, иепно-гомотопное тождественному и такое, что $v(\varphi(x))>v(x)$ для всех ненулевых $x \in C_{i}$ npu $i \leqslant n$.

\section{Список литературы}

[1] С. П. Новиков, "Многозначные функции и функционалы. Аналог теории Морса", Докл. АН СССР, 260 (1981), 31-35; англ. пер.: S. P. Novikov, "Multivalued functions and functionals. An analogue of the Morse theory", Soviet Math. Dokl., 24 (1981), 222-226.

[2] С. П. Новиков, "Гамильтонов формализм и многозначный аналог теории Морса", УМН, 37:5 (1982), 3-49; англ. пер.: S. P. Novikov, "The Hamiltonian formalism and a multi-valued analogue of Morse theory", Russian Math. Surveys, 37:5 (1982), 1-56.

[3] С. П. Новиков, "Блоховские гомологии. Критические точки функций и замкнутых 1-форм", Докл. АН ССCP, 287 (1986), 1321-1324; англ. пер.: S. P. Novikov, "Bloch homology. Critical points of functions and closed 1-forms", Soviet Math. Dokl., 33 (1986), 551-555.

[4] С. П. Новиков, И. Шмельцер, "Периодические решения уравнений Кирхгофа для свободного движения твердого тела в жидкости и расширенная теория Люстерника-Шнирельмана-Морса (ЛШМ). I", Функи. анализ и его прил., 15:3 (1981), 54-66; англ. пер.: S. P. Novikov, I. Smel'tser, "Periodic solutions of the Kirchhoff's equations for the free motion of a rigid body in a fluid and the extended theory of Lyusternik-Shnirel'man-Morse theory (LSM). I", Funct. Anal. Appl., 15:3 (1981), 197-207.

[5] M. Farber, Topology of closed one-forms, Math. Surveys Monogr., 108, Amer. Math. Soc., Providence, RI, 2004.

[6] М. Фарбер, Д. Шютц, “Замкнутые 1-формы в топологии и динамике”, УМH, 63:6 (2008), 91-156; англ. пер.: B. Farber, D. Schutz, "Closed 1-forms in topology and dynamics", Russian Math. Surveys, 63:6 (2008), 1079-1139.

[7] J.-Cl. Sikorav, Points fixes de difféomorphismes symplectiques, intersections de sous-variétés lagrangiennes, et singularités de un-formes fermées, Thèse de Doctorat d'Etat ès Sciences Mathématiques, Université Paris-Sud, Centre d'Orsay, 1987.

[8] R. Bieri, W.D. Neumann, R. Strebel, "A geometric invariant of discrete groups", Invent. Math., 90:3 (1987), 451-477.

[9] R. Bieri, B. Renz, "Valuations on free resolutions and higher geometric invariants of groups", Comment. Math. Helv., 63:3 (1988), 464-497.

[10] M. Farber, "Zeros of closed 1-forms, homoclinic orbits and Lusternik-Schnirelman theory", Topol. Methods Nonlinear Anal., 19:1 (2002), 123-152.

[11] F. Latour, "Existence de 1-formes fermées non singulières dans une classe de cohomologie de de Rham", Publ. Math. Inst. Hautes Ètudes Sci., 80 (1994), 135-194.

[12] M. Farber, "Lusternik-Schnirelman theory and dynamics", Lusternik-Schnirelmann category and related topics (South Hadley, MA, 2001), Contemp. Math., 316, Amer. Math. Soc., Providence, RI, 2002, 95-111. 
[13] М. Фарбер, Т. Каппелер, “Теория Люстерника-Шнирельмана и динамика. II”, Геометрическая топология и теория множеств: K 100-летию со дня рожд. проф. Л.В. Келдыш: Сб. ст., Тр. МИАН, 247, 2004, 252-266; англ. пер.: M. Farber, T. Kappeler, "Lusternik-Schnirelman theory and dynamics. II", Proc. Steklov Inst. Math., 2004, № 4, 232-245.

[14] M. Bestvina, N. Brady, "Morse theory and finiteness properties of groups", Invent. Math., 129:3 (1997), 445-470.

[15] K.S. Brown, Cohomology of groups, Grad. Texts in Math., 87, Springer, New York-Berlin, 1982.

[16] R. Geoghegan, Topological methods in group theory, Grad. Texts in Math., 243, Springer, New York, 2008.

[17] J. Meier, H. Meinert, L. van Wyk, "Higher generation subgroup sets and the इ-invariants of graph groups", Comment. Math. Helv., 73:1 (1998), 22-44.

[18] R. Bieri, R. Geoghegan, D. Kochloukova, The Sigma invariants of Thompson's group F, arXiv: 0807.5138, 2008.

[19] R. Bieri, "Finiteness length and connectivity length for groups", Geometric group theory down under (Canberra, 1996), de Gruyter, Berlin, 1999, 9-22.

[20] R. Bieri, R. Geoghegan, "Connectivity properties of group actions on non-positively curved spaces", Mem. Amer. Math. Soc., 161:765 (2003).

[21] J. Harlander, D. Kochloukova, "The $\Sigma^{3}$-conjecture for metabelian groups", J. London Math. Soc. (2), 67:3 (2003), 609-625.

[22] M. Farber, D. Schütz, "Moving homology classes to infinity", Forum Math., 19:2 (2007), 281-296.

[23] Э. Спеньер, Алгебраическал топология, Мир, М., 1971; англ. пер.: Е. Н. Spanier, Algebraic topology, McGraw-Hill, New York, 1966.

[24] D. Schütz, "Finite domination, Novikov homology and nonsingular closed 1-forms", Math. Z., 252:3 (2006), 623-654.

[25] C. T. C. Wall, "Finiteness conditions for CW-complexes", Ann. of Math. (2), 81:1 (1965), 56-69.

[26] C. T. C. Wall, "Finiteness conditions for CW-complexes. II", Proc. Roy. Soc. Ser. A, 295:1441 (1966), 129-139.

[27] A. Ranicki, "The algebraic theory of finiteness obstruction", Math. Scand., 57:1 (1985), 105-126.

[28] M. Farber, D. Schütz, "Homological category weights and estimates for $\operatorname{cat}^{1}(X, \xi)$ ", J. Eur. Math. Soc. (JEMS), 10:1 (2008), 243-266.

[29] M. Usher, Spectral numbers in Floer theories, arXiv: 0709.1127, 2007.

[30] M. Farber, D. Schütz, "Cohomological estimates for cat $(X, \xi)$ ", Geom. Topol., 11 (2007), 1255-1288.

[31] K.S. Brown, "Homological criteria for finiteness", Comment. Math. Helv., 50:1 (1975), 129-135.

[32] K. S. Brown, "Finiteness properties of groups", Proceedings of the Northwestern conference on cohomology of groups, J. Pure Appl. Algebra, 44:1-3 (1987), 45-75.

М. Фарбер (M. Farber)

University of Durham, UK

E-mail: Michael.Farber@durham.ac.uk

P. Гейган (R. Geoghegan)

State University of New York, Binghamton, USA

E-mail: ross@math.binghamton.edu

\section{Д. Шютц (D. Schütz)}

University of Durham, UK

E-mail: dirk.schuetz@durham.ac.uk
Поступила в редакцию 06.10 .2008 